\title{
Universiteit
}

Leiden

The Netherlands

\section{Construction of the incipient infinite cluster for spread-out oriented percolation above $4+1$ dimensions}

Hofstad, R.; Hollander, W.T.F. den; Slade, G.

\section{Citation}

Hofstad, R., Hollander, W. T. F. den, \& Slade, G. (2002). Construction of the incipient infinite cluster for spread-out oriented percolation above $4+1$ dimensions. Communications In Mathematical Physics, 231(3), 435-461. doi:10.1007/s00220-002-0728-x

Version: $\quad$ Not Applicable (or Unknown)

License: $\quad$ Leiden University Non-exclusive license

Downloaded from: https://hdl.handle.net/1887/61975

Note: To cite this publication please use the final published version (if applicable). 


\title{
Construction of the incipient infinite cluster for spread-out oriented percolation above $4+1$ dimensions
}

\author{
Remco van der Hofstad * \\ Frank den Hollander ${ }^{\dagger}$ \\ Gordon Slade $\ddagger$
}

July 11, 2002

\begin{abstract}
We construct the incipient infinite cluster measure (IIC) for sufficiently spread-out oriented percolation on $\mathbb{Z}^{d} \times \mathbb{Z}_{+}$, for $d+1>4+1$. We consider two different constructions. For the first construction, we define $\mathbb{P}_{n}(E)$ by taking the probability of the intersection of an event $E$ with the event that the origin is connected to $(x, n) \in \mathbb{Z}^{d} \times \mathbb{Z}_{+}$, summing this probability over $x \in \mathbb{Z}^{d}$, and normalising the sum to get a probability measure. We let $n \rightarrow \infty$ and prove existence of a limiting measure $\mathbb{P}_{\infty}$, the IIC. For the second construction, we condition the connected cluster of the origin in critical oriented percolation to survive to time $n$, and let $n \rightarrow \infty$. Under the assumption that the critical survival probability is asymptotic to a multiple of $n^{-1}$, we prove existence of a limiting measure $\mathbb{Q}_{\infty}$, with $\mathbb{Q}_{\infty}=\mathbb{P}_{\infty}$. In addition, we study the asymptotic behaviour of the size of the level set of the cluster of the origin, and the dimension of the cluster of the origin, under $\mathbb{P}_{\infty}$. Our methods involve minor extensions of the lace expansion methods used in a previous paper to relate critical oriented percolation to super-Brownian motion, for $d+1>4+1$.
\end{abstract}

\section{Introduction and results}

\subsection{The incipient infinite cluster}

For oriented percolation on $\mathbb{Z}^{d} \times \mathbb{Z}_{+}$, it was shown in $[3,10]$ that there is no infinite cluster at the critical point. For non-oriented percolation on $\mathbb{Z}^{d}$, proofs that there is no percolation at the critical point are restricted to 2-dimensional and high-dimensional models, and a general proof has remained an elusive goal. The notion of the incipient infinite percolation cluster (IIC) is an

${ }^{*}$ Department of Applied Mathematics, Delft University of Technology, Mekelweg 4, 2628 CD Delft, The Netherlands. Present address: Department of Mathematics and Computer Science, Eindhoven University of Technology, P.O. Box 513, 5600 MB Eindhoven, The Netherlands. rhof stad@win.tue.nl

${ }^{\dagger}$ EURANDOM, P.O. Box 513, 5600 MB Eindhoven, The Netherlands. denhollander@eurandom.tue.nl

${ }^{\ddagger}$ Department of Mathematics, University of British Columbia, Vancouver, BC V6T 1Z2, Canada. slade@math.ubc.ca 
attempt to describe the infinite structure that is emerging but not quite present at the critical point. Various aspects of the IIC are discussed in [1]. There is currently no existence theory for the IIC that is applicable in general dimensions, neither in the oriented nor in the non-oriented setting.

For bond percolation on $\mathbb{Z}^{2}$, Kesten [17] constructed the IIC as a measure on bond configurations in which the origin is almost surely connected to infinity. He gave two different constructions, both leading to the same measure. One construction involved conditioning on the event that the origin is connected to infinity, with bond density $p$ greater than the critical value $p_{c}$, and taking the limit $p \downarrow p_{c}$. Another construction involved conditioning on the event that the origin is connected to the boundary of a box of radius $n$, with $p=p_{c}$, and letting $n \rightarrow \infty$. More recently, Járai $[15,16]$ has shown that several other definitions of the IIC on $\mathbb{Z}^{2}$ yield the same measure as Kesten's. These include the inhomogeneous model of [8], and definitions in terms of invasion percolation [7], the largest cluster in a large box [5], and spanning clusters [1]. The incipient infinite cluster is thus a natural and robust object that can be constructed in many different ways.

No construction of the IIC, as a measure on bond configurations, has been given for any finitedimensional lattice in dimensions greater than 2. In the present paper, we consider sufficiently spread-out oriented percolation on $\mathbb{Z}^{d} \times \mathbb{Z}_{+}$, with $d+1>4+1$, and propose two definitions of the IIC.

Perhaps the most natural definition of the IIC for oriented percolation is the measure $\mathbb{Q}_{\infty}$ obtained by conditioning the cluster of the origin to survive to time $n$, with $p=p_{c}$, and then letting $n \rightarrow \infty$. Of course, it is not obvious that the limit exists.

For another possible definition, we set $p=p_{c}$ and define $\mathbb{P}_{n}(E)$ by taking the probability of the intersection of an event $E$ with the event that the origin is connected to $(x, n) \in \mathbb{Z}^{d} \times \mathbb{Z}_{+}$, summing this probability over $x \in \mathbb{Z}^{d}$, and normalising the sum to get a probability measure. We will let $n \rightarrow \infty$ and prove existence of a limiting measure $\mathbb{P}_{\infty}$. It is clear from the definition that $\mathbb{P}_{\infty}$ will be supported on configurations in which the origin is connected to infinity.

In view of the apparent robustness of the IIC, it is natural to expect that $\mathbb{P}_{\infty}=\mathbb{Q}_{\infty}$. In fact, we will prove that $\mathbb{Q}_{\infty}$ exists and equals $\mathbb{P}_{\infty}$, subject to the assumption that the critical survival probability behaves asymptotically as a multiple of $n^{-1}$. We believe that the methods of [14] can be adapted to prove this assumption, and we plan to return to this problem in a future publication [12]. Our constructions are restricted to $d+1>4+1$ due to the appearance in proofs of Feynman diagrams that require $d>4$ for convergence, as in [14, 20, 21].

Finally, we will derive various properties of the IIC measure $\mathbb{P}_{\infty}$. These include statements that under $\mathbb{P}_{\infty}$ the cluster of the origin is infinite, the number of particles in the cluster of the origin at time $m$ grows like $m$ times a size-biased exponential random variable, and the cluster has a 4-dimensional character.

An alternate approach to the IIC is via a scaling limit. For oriented percolation, the goal is to understand the distribution of critical clusters that survive to time $n$, with the lattice spacing shrinking as an appropriate power of $n$, in the limit $n \rightarrow \infty$. Such a program was carried out in [14], where it was shown that the scaling limit for sufficiently spread-out oriented percolation above the upper critical dimension $4+1$ is intimately related to super-Brownian motion. (Related results for non-oriented percolation were obtained in [11].) This suggests that large critical clusters are closely related to large critical branching random walk clusters, for $d+1>4+1$. The results and methods in the present paper are based on minor extensions of the results and lace expansion techniques used in [14]. The lace expansion was first applied to oriented percolation by Nguyen 
and Yang $[20,21]$.

\subsection{Existence of the IIC measure}

The spread-out oriented percolation models are defined as follows. Consider the graph with vertices $\mathbb{Z}^{d} \times \mathbb{Z}_{+}$and directed bonds $((x, n),(y, n+1))$, for $n \geq 0$ and $x, y \in \mathbb{Z}^{d}$. Let $D: \mathbb{Z}^{d} \rightarrow[0,1]$ be a fixed function. Let $p \in\left[0,\|D\|_{\infty}^{-1}\right]$, where $\|\cdot\|_{\infty}$ denotes the supremum norm, so that $p D(x) \leq 1$ for all $x$. We associate to each directed bond $((x, n),(y, n+1))$ an independent random variable taking the value 1 with probability $p D(y-x)$ and 0 with probability $1-p D(y-x)$. We say a bond is occupied when the corresponding random variable is 1 , and vacant when the random variable is 0 . Given a configuration of occupied bonds, we say that $(x, n)$ is connected to $(y, m)$, and write $(x, n) \longrightarrow(y, m)$, if there is an oriented path from $(x, n)$ to $(y, m)$ consisting of occupied bonds, or if $(x, n)=(y, m)$. The joint probability distribution of the bond variables will be denoted $\mathbb{P}$, with corresponding expectation denoted $\mathbb{E}$. Note that $p$ is not a probability. We will always work at the critical percolation threshold, i.e., at $p=p_{c}$, and omit subscripts $p_{c}$ from the notation.

A simple example is

$$
D(x)= \begin{cases}(2 L+1)^{-d} & \|x\|_{\infty} \leq L \\ 0 & \text { otherwise }\end{cases}
$$

for which bonds are of the form $((x, n),(y, n+1))$ with $\|x-y\|_{\infty} \leq L$, and a bond is occupied with probability $p(2 L+1)^{-d}$. In this parametrisation, $p_{c}$ tends to 1 as $L \rightarrow \infty$.

Our results hold for any function $D$ that obeys the assumptions listed in [14, Section 1.2]. These assumptions involve a positive parameter $L$ which serves to spread out the connections, and which we will take to be large. In particular, they require that $\sum_{x \in \mathbb{Z}^{d}} D(x)=1$, that $D(x) \leq C L^{-d}$ for all $x$, and, with $\sigma$ defined by

$$
\sigma^{2}=\sum_{x \in \mathbb{Z}^{d}}|x|^{2} D(x)
$$

where $|\cdot|$ denotes the Euclidean norm on $\mathbb{R}^{d}$, that $C_{1} L \leq \sigma \leq C_{2} L$. Full details regarding the assumptions can be found in [14]. The function defined by (1.1) does obey the assumptions.

Let $\mathcal{F}$ denote the $\sigma$-algebra of events. A cylinder event is an event that is determined by the occupation status of a finite set of bonds. We denote the algebra of cylinder events by $\mathcal{F}_{0}$. Then $\mathcal{F}$ is the $\sigma$-algebra generated by $\mathcal{F}_{0}$. For our first definition of the IIC, we begin by defining $\mathbb{P}_{n}$ by

$$
\mathbb{P}_{n}(E)=\frac{1}{\tau_{n}} \sum_{x \in \mathbb{Z}^{d}} \mathbb{P}(E \cap\{(0,0) \longrightarrow(x, n)\}) \quad\left(E \in \mathcal{F}_{0}\right),
$$

where $\tau_{n}=\sum_{x \in \mathbb{Z}^{d}} \tau_{n}(x)$ with $\tau_{n}(x)=\mathbb{P}((0,0) \longrightarrow(x, n))$. We then define $\mathbb{P}_{\infty}$ by setting

$$
\mathbb{P}_{\infty}(E)=\lim _{n \rightarrow \infty} \mathbb{P}_{n}(E) \quad\left(E \in \mathcal{F}_{0}\right),
$$

assuming the limit exists. The following theorem shows that this definition produces a probability measure on $\mathcal{F}$ under which the origin is almost surely connected to infinity.

Theorem 1.1. Let $d+1>4+1$ and $p=p_{c}$. There is an $L_{0}=L_{0}(d)$ such that for $L \geq L_{0}$, the limit in (1.4) exists for every cylinder event $E \in \mathcal{F}_{0}$. Moreover, $\mathbb{P}_{\infty}$ extends to a probability measure on the $\sigma$-algebra $\mathcal{F}$, and the origin is almost surely connected to infinity under $\mathbb{P}_{\infty}$. 
Let

$$
S_{n}=\{(0,0) \longrightarrow n\}=\left\{(0,0) \longrightarrow(x, n) \text { for some } x \in \mathbb{Z}^{d}\right\}
$$

denote the event that the cluster of the origin survives to time $n$. For our second definition of the IIC, we begin by defining $\mathbb{Q}_{n}$ by

$$
\mathbb{Q}_{n}(E)=\mathbb{P}\left(E \mid S_{n}\right) \quad\left(E \in \mathcal{F}_{0}\right) .
$$

We then define $\mathbb{Q}_{\infty}$ by setting

$$
\mathbb{Q}_{\infty}(E)=\lim _{n \rightarrow \infty} \mathbb{Q}_{n}(E) \quad\left(E \in \mathcal{F}_{0}\right)
$$

assuming the limit exists.

Not surprisingly, the existence of $\mathbb{Q}_{\infty}$ turns out to be related to the asymptotic behaviour of the critical survival probability

$$
\theta_{n}=\mathbb{P}\left(S_{n}\right)
$$

We will assume that for critical spread-out oriented percolation with $d+1>4+1$ and $L$ sufficiently large, there is a finite positive constant $B$ such that

$$
\lim _{n \rightarrow \infty} n \theta_{n}=1 / B
$$

Although there is currently no proof of (1.9), we intend to return to this question in a future publication [12]. Assuming (1.9), the following theorem gives existence of the IIC measure $\mathbb{Q}_{\infty}$, with $\mathbb{Q}_{\infty}=\mathbb{P}_{\infty}$.

Theorem 1.2. Let $d+1>4+1$ and $p=p_{c}$, and assume (1.9). There is an $L_{0}=L_{0}(d)$ such that for $L \geq L_{0}$, the limit in (1.7) exists for every cylinder event $E \in \mathcal{F}_{0}$. Moreover, $\mathbb{Q}_{\infty}$ extends to a probability measure on the $\sigma$-algebra $\mathcal{F}$, and $\mathbb{Q}_{\infty}=\mathbb{P}_{\infty}$.

We conjecture that the measure $\mathbb{P}_{n}^{(x)}$ defined by

$$
\mathbb{P}_{n}^{(x)}(E)=\frac{1}{\tau_{n}(x)} \mathbb{P}(E \cap\{(0,0) \longrightarrow(x, n)\})
$$

converges to the IIC measure $\mathbb{P}_{\infty}$ of Theorem 1.1 , for each fixed $x \in \mathbb{Z}^{d}$. We are not able to prove this, without some strengthening of the local central limit theorem of $[13,14]$. Some intuition that supports both this conjecture and the conjecture that $\mathbb{P}_{\infty}=\mathbb{Q}_{\infty}$, in general dimensions, is given near the beginning of Section 3.1.

Of the possible definitions of the incipient infinite cluster for oriented percolation, we find $\mathbb{P}_{\infty}$ the easiest to work with and the most closely related to the work of [14] connecting critical oriented percolation and super-Brownian motion. For example, if we let $E$ be the event that $(0,0) \longrightarrow\left(y_{i}, m_{i}\right)(i=1, \ldots, s)$, then the right side of (1.3) involves the probability that the origin is connected to $(x, n)$, as well as to $\left(y_{i}, m_{i}\right)(i=1, \ldots, s)$. The scaling of such $(s+2)$-point functions was shown in [14] to be described by related quantities for the canonical measure of super-Brownian motion, for $d>4, p=p_{c}$, and $L$ sufficiently large. We will use this scaling in establishing the properties of the IIC measure stated in the following section.

The asymptotic formula (1.9) is belived to fail in low dimensions, and our methods do not apply at all for $d \leq 4$. Nevertheless, we expect that $\mathbb{P}_{\infty}$ and $\mathbb{Q}_{\infty}$ exist and are equal in all dimensions. 


\subsection{Properties of the IIC measure}

The Hausdorff dimension of the connected cluster of the origin under the IIC is believed to equal 4 almost surely, for $d+1>4+1$. The following theorem provides a weaker statement, indicating a 4-dimensional aspect to the IIC. In order to be able to state the result, we let

$$
C(0,0)=\left\{(y, m) \in \mathbb{Z}^{d} \times \mathbb{Z}_{+}:(0,0) \longrightarrow(y, m)\right\}
$$

denote the connected cluster of the origin, and let

$$
D_{R}=\mathbb{E}_{\infty}[\#\{(y, m) \in C(0,0):|y| \leq R\}]
$$

denote the expected number of sites in the cluster of the origin that are at most a distance $R$ away from the origin, under $\mathbb{P}_{\infty}$.

Theorem 1.3. Let $d+1>4+1$ and $p=p_{c}$. There are $L_{0}=L_{0}(d)$ and $C_{i}=C_{i}(L, d)>0$ such that for $L \geq L_{0}$,

$$
C_{1} R^{4} \leq D_{R} \leq C_{2} R^{4}
$$

In Section 5.1, where Theorem 1.3 is proved, we will also define the $r$-point functions of $\mathbb{P}_{\infty}$ and obtain results concerning their asymptotic behaviour.

For our next property of $\mathbb{P}_{\infty}$, we let

$$
N_{m}=\#\left\{y \in \mathbb{Z}^{d}:(0,0) \longrightarrow(y, m)\right\}
$$

denote the number of sites at time $m$ to which the origin is connected. We recall that the size-biased exponential random variable with parameter $\lambda$ has density

$$
f(x)=\lambda^{2} x e^{-\lambda x} \quad(x \geq 0) .
$$

The following theorems describe the distribution of $N_{m}$ under $\mathbb{P}_{\infty}$ and $\mathbb{Q}_{m}$. The constants $A$ and $V$ appearing in their statements are finite positive constants arising in the scaling of the 2- and 3point functions [14] (see Theorem 4.1 below), while $B$ is the constant in (1.9). The three constants $A, V, B$ depend on $d$ and $L$.

Theorem 1.4. Let $d+1>4+1$ and $p=p_{c}$. There is an $L_{0}=L_{0}(d)$ such that for $L \geq L_{0}$,

$$
\lim _{m \rightarrow \infty} \mathbb{E}_{\infty}\left[\left(\frac{N_{m}}{m}\right)^{l}\right]=\left(\frac{A^{2} V}{2}\right)^{l}(l+1) ! \quad(l=1,2, \ldots) .
$$

Consequently, under $\mathbb{P}_{\infty}, m^{-1} N_{m}$ converges weakly to a size-biased exponential random variable with parameter $\lambda=2 /\left(A^{2} V\right)$.

Theorem 1.5. Let $d+1>4+1$ and $p=p_{c}$. Assume that (1.9) holds. Then

$$
B=\frac{A V}{2} \text {. }
$$

In addition, there is an $L_{0}=L_{0}(d)$ such that for $L \geq L_{0}$,

$$
\lim _{m \rightarrow \infty} \mathbb{E}_{\mathbb{Q}_{m}}\left[\left(\frac{N_{m}}{m}\right)^{l}\right]=\left(\frac{A^{2} V}{2}\right)^{l} l ! \quad(l=1,2, \ldots) .
$$

Consequently, under $\mathbb{Q}_{m}, m^{-1} N_{m}$ converges weakly to an exponential random variable with parameter $\lambda=2 /\left(A^{2} V\right)$. 
The identity (1.17), which holds under the assumption (1.9), expresses a relation between the three constants $B, A$ and $V$. It is shown in [14] that $A$ and $V$ both equal $1+\mathcal{O}\left(L^{-d}\right)$, and hence (1.17) implies that $B=\frac{1}{2}+\mathcal{O}\left(L^{-d}\right)$.

Under the assumption that (1.9) holds, it follows from Theorems 1.4-1.5 that $m^{-1} N_{m}$ converges to a size-biased exponential random variable under $\mathbb{Q}_{\infty}=\mathbb{P}_{\infty}$, and to an exponential random variable under $\mathbb{Q}_{m}$. A similar contrast can be proved for the behaviour of $m^{-1} N_{m}$ for critical branching random walk (in general dimensions, with an offspring distribution with finite variance), where again the size-biased exponential distribution occurs when the branching random walk is conditioned to survive to infinite time, and the exponential distribution occurs when the branching random walk is conditioned to survive until time $m$. This is consistent with the general philosophy that oriented percolation behaves like branching random walk above the upper critical dimension $4+1$, as already noted at the end of Section 1.1 .

Finally, we remark that we will give a formula for $\mathbb{P}_{\infty}(E)$ in terms of the lace expansion in (2.29) below, when $E \in \mathcal{F}_{0}$ is a cylinder event.

\subsection{Organisation}

The remainder of this paper is organised as follows. In Section 2 we prove Theorem 1.1, and in Section 3 we prove Theorem 1.2. In Section 4, we recall the main result of [14] linking critical oriented percolation and super-Brownian motion, and derive some elementary properties of the moment measures of the canonical measure of super-Brownian motion. Using the results of Section 4, we then prove Theorems 1.3-1.5 in Section 5.

\section{Proof of Theorem 1.1}

The proof of Theorem 1.1 uses a modification of the Nguyen-Yang lace expansion for oriented percolation $[20,21]$ (see also [14, Section 3]), to derive an expansion for $\mathbb{P}_{n}(E)$ of $(1.3)$. We derive the modified expansion in Section 2.1, and use it to prove Theorem 1.1 in Section 2.2.

\subsection{The lace expansion for $\mathbb{P}_{n}$}

Throughout this section, we fix $p \in\left[0,\|D\|_{\infty}^{-1}\right]$ and $m \geq 1$.

A cylinder event $E$ is an event that depends on the occupation status of a finite set of bonds $\mathcal{B}(E)$. Let $\mathcal{E}_{m}$ denote the set of cylinder events $E$ for which the maximum time appearing in $\mathcal{B}(E)$

is $m$, and fix $E \in \mathcal{E}_{m}$. Given a bond configuration, we say that a bond $b$ is pivotal for an increasing event $F$ if $F$ occurs when $b$ is made to be occupied and $F$ does not occur when $b$ is made to be vacant. For $E \in \mathcal{E}_{m}, n \geq m$ and $0 \leq t \leq n$, we define

$$
\begin{aligned}
\tau_{n, t}(x ; E) & =\mathbb{P}(E \cap\{(0,0) \longrightarrow(x, n) \text { with exactly } t \text { occupied pivotal bonds }\}), \\
\tau_{n}(x ; E) & =\mathbb{P}(E \cap\{(0,0) \longrightarrow(x, n)\})=\sum_{t=0}^{n} \tau_{n, t}(x ; E),
\end{aligned}
$$

where the pivotal bonds are pivotal for the event $F=\{(0,0) \longrightarrow(x, n)\}$. Then (1.3) reads

$$
\mathbb{P}_{n}(E)=\frac{1}{\tau_{n}} \sum_{x \in \mathbb{Z}^{d}} \tau_{n}(x ; E) .
$$


We write $(x, n) \Longrightarrow(y, m)$ to denote the event that $(x, n)$ is doubly-connected to $(y, m)$, i.e., the event that there exist at least two bond-disjoint occupied paths from $(x, n)$ to $(y, m)$, or $(x, n)=(y, m)$. Given a bond $b=((x, n),(y, n+1))$, let $\bar{b}=(y, n+1)$ be the "top" of $b$, and $\underline{b}=(x, n)$ be the "bottom" of $b$. We will write $\bar{b}<\bar{b}^{\prime}$ to mean that the temporal component of $\bar{b}$ is less than that of $\bar{b}^{\prime}$, and, in an abuse of notation, we write $\bar{b} \leq n$ when the temporal component of $\bar{b}$ is less than or equal to $n$. For $t \geq 1$, let

$$
B_{t}(n)=\left\{\vec{b}=\left(b_{1}, \ldots, b_{t}\right): 0<\bar{b}_{1}<\cdots<\bar{b}_{t} \leq n\right\}
$$

denote the ordered vectors of $t$ bonds, between times 0 and $n$. Given $x \in \mathbb{Z}^{d}$ and $\vec{b} \in B_{t}(n)$, we define $\bar{b}_{0}=0, \underline{b}_{t+1}=(x, n)$, and

$$
T_{t}(\vec{b},(x, n))= \begin{cases}\{(0,0) \Longrightarrow(x, n)\} & (t=0) \\ \bigcap_{i=1}^{t}\left\{b_{i} \text { occupied }\right\} \bigcap_{j=0}^{t}\left\{\bar{b}_{j} \Longrightarrow \underline{b}_{j+1}\right\} & (1 \leq t \leq n) .\end{cases}
$$

Note that if $T_{t}(\vec{b},(x, n))$ occurs, then the only possible candidates for occupied pivotal bonds for the event $(0,0) \rightarrow(x, n)$ are the elements of $\vec{b}$.

For $0 \leq s<t$, we define the random variables

$$
K[s, t]=\prod_{s \leq i<j \leq t}\left(1+U_{i j}\right), \quad U_{i j}=-I\left[\bar{b}_{i} \Longrightarrow \underline{b}_{j+1}\right]
$$

and we set $K[s, s]=K[s+1, s]=1$. The product in $(2.6)$ is 0 or 1 . If $K[0, t]=1$ and $T_{t}(\vec{b},(x, n))$ occurs, then the occupied pivotal bonds for the event $(0,0) \rightarrow(x, n)$ are precisely the elements of $\vec{b}$. Therefore (2.1) becomes

$$
\tau_{n, t}(x ; E)= \begin{cases}\mathbb{P}(E \cap\{(0,0) \Longrightarrow(x, n)\}) & (t=0) \\ \sum_{\vec{b} \in B_{t}(n)} \mathbb{E}\left[I[E] I\left[T_{t}(\vec{b},(x, n))\right] K[0, t]\right] & (1 \leq t \leq n) .\end{cases}
$$

The identity (2.7) can be understood by regarding the cluster of the origin as a "string of sausages" as depicted in Figure 1, where the "string" is specified by the bonds $\vec{b}$. The event $E$ occurs before time $m$.

The lace expansion involves a decomposition of $K[0, t]$. To describe this, we need some standard terminology $[6,19]$. A graph on an interval $[s, t]$ is a set $\Gamma=\left\{i_{1} j_{1}, \ldots, i_{M} j_{M}\right\}$ of edges, with $s \leq i_{l}<j_{l} \leq t$ for each $l$. We say that a graph $\Gamma$ is connected on $[s, t]$ if $\bigcup_{i j \in \Gamma}[i, j]=[s, t]$. We denote the set of connected graphs on $[s, t]$ by $\mathcal{G}[s, t]$, and let

$$
J[s, t]=\sum_{\Gamma \in \mathcal{G}[s, t]} \prod_{i j \in \Gamma} U_{i j} .
$$

We set $J[0,0]=1$. Expansion of the product in (2.6) gives a sum over all graphs, and a partition of this sum according to the support of the connected component of $m$ leads to the decomposition

$$
K[0, t]=\sum_{s=0}^{t} M[0, s ; m] K[s+1, t] \quad(m \in[0, n] \text { fixed, } t \geq 0),
$$




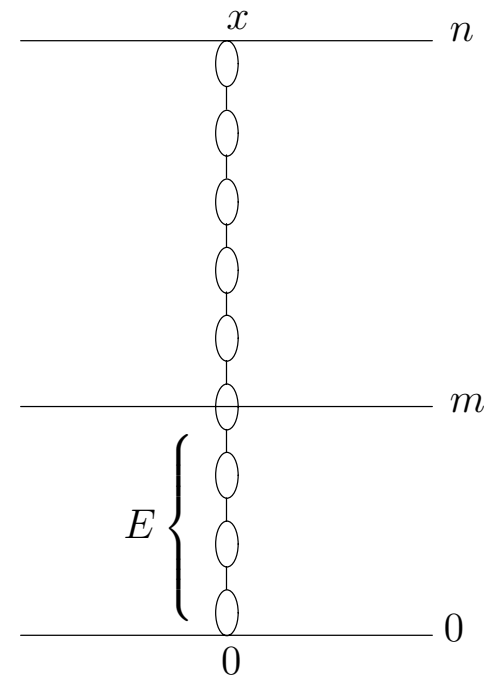

Figure 1: Schematic depiction of a configuration contributing to $\tau_{n}(x ; E)$ as a "string of sausages." The event $E \in \mathcal{E}_{m}$ is required to occur.

where

$$
M[0, s ; m]=\sum_{i=0}^{s} K[0, i-1] J[i, s] I\left[\bar{b}_{i} \leq m \leq \underline{b}_{s+1}\right] .
$$

See $[24,(2.10)]$ or $[19$, Lemma 5.2.5] for more details on (2.9)-(2.10) in the case of a slightly different definition of graph connectivity. For $l \geq m$, we define

$$
\varphi_{l, s}(v ; E)= \begin{cases}\mathbb{P}(E \cap\{(0,0) \Longrightarrow(v, l)\}) & (s=0) \\ \sum_{\vec{b} \in B_{s}(l)} \mathbb{E}\left[I[E] I\left[T_{s}(\vec{b},(v, l))\right] M[0, s ; m]\right] & (1 \leq s \leq l)\end{cases}
$$

with $\underline{b}_{s+1}=(v, l)$, and

$$
\varphi_{l}(E)=\sum_{v \in \mathbb{Z}^{d}} \sum_{s=0}^{l} \varphi_{l, s}(v ; E) .
$$

Although it is not explicit in the notation, $\varphi_{l, s}(v ; E)$ and $\varphi_{l}(E)$ depend on $m$ by definition. In particular, we are restricting to $E \in \mathcal{E}_{m}$.

The following lemma relates $\tau_{n, t}(x ; E)$ and $\varphi_{l, s}(u ; E)$.

Lemma 2.1. For $E \in \mathcal{E}_{m}, n \geq m$, and $0 \leq t \leq n$,

$$
\tau_{n, t}(x ; E)=\sum_{(u, v)} \sum_{l=m}^{n-1} \sum_{s=0}^{t-1} \varphi_{l, s}(u ; E) p D(v-u) \tau_{n-l-1, t-s-1}(x-v)+\varphi_{n, t}(x ; E),
$$

where the first term on the right side is interpreted as zero when $t=0$.

Proof. The proof is a standard lace expansion argument. For $t=0$, (2.13) follows immediately from (2.7) and (2.11). For $t \geq 1$, we substitute (2.9) into (2.7). The $s=t$ term of (2.9) gives rise to the second term on the right side of (2.13). It therefore remains to show that

$$
\sum_{\vec{b} \in B_{t}(n)} \sum_{s=0}^{t-1} \mathbb{E}\left[I[E] I\left[T_{t}(\vec{b},(x, n))\right] M[0, s ; m] K[s+1, t]\right]
$$


is equal to the first term on the right side of (2.13). For this, given $\vec{b}$ and $s$, we decompose the random variables appearing in (2.14) into the three factors:

$$
\begin{gathered}
I[E] I\left[\cap_{r=1}^{s}\left\{b_{r} \text { occupied }\right\} \cap_{r=0}^{s}\left\{\bar{b}_{r} \Longrightarrow \underline{b}_{r+1}\right\}\right] M[0, s ; m], \\
\left\{b_{s+1} \text { occupied }\right\}, \\
I\left[\cap_{r=s+2}^{t}\left\{b_{r} \text { occupied }\right\} \cap_{r=s+1}^{t}\left\{\bar{b}_{r} \Longrightarrow \underline{b}_{r+1}\right\}\right] K[s+1, t] .
\end{gathered}
$$

These random variables depend on bonds below $\underline{b}_{s+1}$, between $\underline{b}_{s+1}$ and $\bar{b}_{s+1}$, and above $\bar{b}_{s+1}$, respectively. Recalling (2.7) and (2.11), we see that the expectation factors to give the first term on the right side of $(2.13)$. In $(2.13), l$ corresponds to the temporal component of $\underline{b}_{s+1}$, while $u$ and $v$ are the lower and upper spatial components of $b_{s+1}$.

Summation over $t=0, \ldots, n$ and $x \in \mathbb{Z}^{d}$ in $(2.13)$ gives

$$
\sum_{x \in \mathbb{Z}^{d}} \tau_{n}(x ; E)=\sum_{l=m}^{n-1} \varphi_{l}(E) p \tau_{n-l-1}+\varphi_{n}(E) .
$$

With (2.3), this gives the expansion

$$
\mathbb{P}_{n}(E)=\frac{1}{\tau_{n}}\left[\sum_{l=m}^{n-1} \varphi_{l}(E) p \tau_{n-l-1}+\varphi_{n}(E)\right] .
$$

Next, we rewrite $\varphi_{l}(E)$ in terms of laces. A lace on $[k, l]$ is an element of $\mathcal{G}[k, l]$ such that the removal of any edge will result in a disconnected graph. Given a connected graph $\Gamma \in \mathcal{G}[k, l]$, we define the lace $\mathrm{L}_{\Gamma} \subset \Gamma$ to be the graph consisting of edges $s_{1} t_{1}, s_{2} t_{2}, \ldots$ given by

$$
\begin{aligned}
t_{1} & =\max \{t: k t \in \Gamma\}, \\
t_{i+1} & =\max \left\{t: \exists s \leq t_{i} \text { such that } s t \in \Gamma\right\}, \quad s_{1}=k,
\end{aligned}
$$

It is not difficult to check that $\mathrm{L}_{\Gamma}$ is indeed a lace. Given a lace $L$, let $\mathcal{C}(L)$ denote the set of compatible edges, i.e., the set of edges $i j$ such that $\mathrm{L}_{L \cup\{i j\}}=L$. Define $\mathcal{L}^{(N)}[k, l]$ to be the set of laces on the interval $[k, l]$ consisting of exactly $N$ edges. It is then a standard fact $[6,19]$ that

$$
J[i, j]=\sum_{N=1}^{\infty}(-1)^{N} J^{(N)}[i, j] \quad(j>i \geq 0),
$$

with

$$
J^{(N)}[i, j]=\sum_{L \in \mathcal{L}^{(N)}[i, j]} \prod_{s t \in L}\left(-U_{s t}\right) \prod_{s^{\prime} t^{\prime} \in \mathcal{C}(L)}\left(1+U_{s^{\prime} t^{\prime}}\right) .
$$

For $l \geq m$, we define

$$
\begin{aligned}
\varphi_{l}^{(0)}(E)= & \sum_{v \in \mathbb{Z}^{d}} \mathbb{P}(E \cap\{(0,0) \Longrightarrow(v, l)\}) \\
& +\sum_{s=1}^{m} \sum_{v \in \mathbb{Z}^{d}} \sum_{\vec{b} \in B_{s}(l)} \mathbb{E}\left(I[E] I\left[T_{s}(\vec{b},(v, l))\right] K[0, s-1] I\left[\bar{b}_{s} \leq m \leq \underline{b}_{s+1}\right]\right)
\end{aligned}
$$


which combines the first line of (2.11) for $s=0$ with the contribution to the second line of (2.11) due to $i=s$ in the definition of $M[0, s ; m]$ in (2.10). (The upper limit of the sum over $s$ in $(2.23)$ can be taken to be $m$ rather than $l$ in $(2.23)$ because the restriction $\bar{b}_{s} \leq m$ can occur only when $s \leq m$.) For $N \geq 1$ and $l \geq m$, we also define

$$
\varphi_{l}^{(N)}(E)=\sum_{s=1}^{l} \sum_{v \in \mathbb{Z}^{d}} \sum_{\vec{b} \in B_{s}(l)} \mathbb{E}\left(I[E] I\left[T_{s}(\vec{b},(v, l))\right] \sum_{i=0}^{s-1} K[0, i-1] J^{(N)}[i, s] I\left[\bar{b}_{i} \leq m \leq \underline{b}_{s+1}\right]\right) .
$$

It follows from (2.10)-(2.12) and (2.21) that

$$
\varphi_{l}(E)=\sum_{N=0}^{\infty}(-1)^{N} \varphi_{l}^{(N)}(E) .
$$

Equations (2.19) and (2.23)-(2.25) constitute the lace expansion for $\mathbb{P}_{n}$.

\subsection{Estimates on the lace expansion for $\mathbb{P}_{n}$}

Throughout this section, we fix $p=p_{c}$. It follows from [14, Theorem 1.1(a)] that, under the hypotheses of Theorem 1.1, there is an $A \in(0, \infty)$ such that

$$
\lim _{n \rightarrow \infty} \tau_{n}=A .
$$

To prove Theorem 1.1, we will use (2.19), (2.26) and the following lemma. We write $\beta=L^{-d}$, and recall from [14] that $p_{c}=1+\mathcal{O}(\beta)$ for $d+1>4+1$.

Lemma 2.2. Let $d+1>4+1, p=p_{c}$ and $E \in \mathcal{E}_{m}$. There are $K=K(d)$ and $L_{0}=L_{0}(d)$ such that for $L \geq L_{0}$,

$$
\left|\varphi_{l}(E)\right| \leq K m \beta(l-m+1)^{-d / 2} \quad(l \geq m+1) .
$$

Proof of Theorem 1.1 subject to Lemma 2.2. Let $E \in \mathcal{E}_{m}$. By (2.19),

$$
\mathbb{P}_{\infty}(E)=\lim _{n \rightarrow \infty} \mathbb{P}_{n}(E)=\lim _{n \rightarrow \infty} \frac{1}{\tau_{n}}\left[\sum_{l=m}^{n-1} \varphi_{l}(E) p_{c} \tau_{n-l-1}+\varphi_{n}(E)\right] .
$$

It therefore follows from (2.26), Lemma 2.2 and the dominated convergence theorem that

$$
\mathbb{P}_{\infty}(E)=p_{c} \sum_{l=m}^{\infty} \varphi_{l}(E) \quad\left(E \in \mathcal{E}_{m}\right) .
$$

This proves existence of and gives a formula for the limit (1.4), for every cylinder event $E \in \mathcal{F}_{0}$.

To complete the proof of Theorem 1.1, it remains to show that $\mathbb{P}_{\infty}$ can be extended to a probability measure on the $\sigma$-algebra $\mathcal{F}$, and that the origin is almost surely connected to infinity under this extension. The extension of $\mathbb{P}_{\infty}$ to $\mathcal{F}$ follows from Kolmogorov's extension theorem (see e.g. [23]), since the consistency hypothesis of Kolmogorov's extension theorem is satisfied by definition of $\mathbb{P}_{n}(E)$ and $\mathbb{P}_{\infty}(E)$ in (1.3)-(1.4). In addition, $\mathbb{P}_{n}((0,0) \longrightarrow N)=1$ for every $n \geq N$, so $\mathbb{P}_{\infty}((0,0) \longrightarrow N)=1$ for every $N \geq 1$, and hence $\mathbb{P}_{\infty}((0,0) \longrightarrow \infty)=\lim _{N \rightarrow \infty} \mathbb{P}_{\infty}((0,0) \longrightarrow$ $N)=1$. 


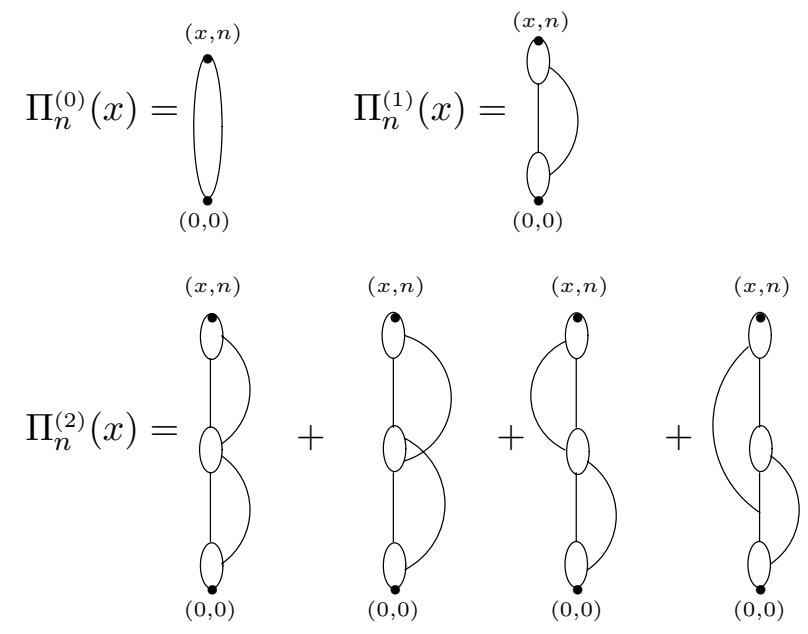

Figure 2: Schematic depiction of disjoint connections required by $\Pi_{n}^{(N)}(x)(N=0,1,2)$.

Proof of Lemma 2.2. Fix $m, E \in \mathcal{E}_{m}$, and $l \geq m+1$. The proof involves a comparison of $\varphi_{l}^{(N)}(E)$ with quantities arising in the Nguyen-Yang lace expansion for the two-point function [20]. We use the notation and results of [14, Sections 3.2 and 4.4]; this notation is not identical to that of [20]. Quantities $\Pi_{n}^{(N)}(x)$ are defined in [14, Sections 3.2] by

$$
\Pi_{n}^{(N)}(x)= \begin{cases}\mathbb{P}((0,0) \Longrightarrow(x, n))-\delta_{x, 0} \delta_{n, 0} & (N=0) \\ \sum_{s=1}^{n} \sum_{\vec{b} \in B_{s}(n)} \mathbb{E}\left[I\left[T_{s}(\vec{b},(x, n))\right] J^{(N)}[0, s]\right] & (N \geq 1) .\end{cases}
$$

Disjoint connections implied by the right side of (2.30) are depicted in Figure 2. We will use the fact, proved in $[14,(4.57)]$, that

$$
\sum_{x \in \mathbb{Z}^{d}} \Pi_{n}^{(N)}(x) \leq C^{N} \beta^{N \vee 1}(n+1)^{-d / 2} \quad(N \geq 0)
$$

assuming the hypotheses of Theorem 1.1. Our assumption that $d>4$ is used only in invoking $(2.31)$.

We consider first the case $N=0$. Recall the definition of $\varphi_{l}^{(0)}(E)$ in $(2.23)$. Because $I[E] \leq$ 1 , the first term on the right side of $(2.23)$ is bounded above by $\sum_{v} \Pi_{l}^{(0)}(v)$, which is at most $C \beta(l+1)^{-d / 2}$ by $(2.31)$. The second term on the right side of $(2.23)$ is bounded above by

$$
\begin{aligned}
& \sum_{s=1}^{m} \sum_{v \in \mathbb{Z}^{d}} \sum_{\vec{b} \in B_{s}(l)} \mathbb{E}\left(I\left[T_{s}(\vec{b},(v, l))\right] K[0, s-1] I\left[\bar{b}_{s} \leq m \leq \underline{b}_{s+1}\right]\right) \\
& \quad=\sum_{w, y, v \in \mathbb{Z}^{d}} \sum_{a=0}^{m-1} \tau_{a}(y) p_{c} D(w-y) \Pi_{l-a-1}^{(0)}(v-w)=\sum_{a=0}^{m-1} \tau_{a} p_{c} \sum_{v \in \mathbb{Z}^{d}} \Pi_{l-a-1}^{(0)}(v),
\end{aligned}
$$

where we have factored the expectation as in the proof of Lemma 2.1, and where $\underline{b}_{s}$ in the first line corresponds to $(y, a)$ in the second line. Therefore, letting $C$ denote a generic constant and using (2.26) and (2.31), we get

$$
\varphi_{l}^{(0)}(E) \leq C \beta(l+1)^{-d / 2}+C \beta \sum_{a=0}^{m-1}(l-a)^{-d / 2} \leq C \beta m(l-m+1)^{-d / 2} .
$$


We next consider the case $N \geq 1$. Applying the inequality $I[E] \leq 1$ in $(2.24)$, we get

$$
\varphi_{l}^{(N)}(E) \leq \sum_{s=1}^{l} \sum_{v \in \mathbb{Z}^{d}} \sum_{\vec{b} \in B_{s}(l)} \mathbb{E}\left(I\left[T_{s}(\vec{b},(v, l))\right] \sum_{i=0}^{s-1} K[0, i-1] J^{(N)}[i, s] I\left[\bar{b}_{i} \leq m \leq \underline{b}_{s+1}\right]\right) .
$$

We may then factor the random variables on the right side into factors depending on bonds below $\underline{b}_{i}$, between $\underline{b}_{i}$ and $\bar{b}_{i}$, and above $\bar{b}_{i}$, respectively, as in the proof of Lemma 2.1. This leads to

$$
\varphi_{l}^{(N)}(E) \leq \sum_{v \in \mathbb{Z}^{d}} \Pi_{l}^{(N)}(v)+\sum_{a=0}^{m-1} \tau_{a} p_{c} \sum_{v \in \mathbb{Z}^{d}} \Pi_{l-a-1}^{(N)}(v) \quad(N \geq 1)
$$

where the terms on the right side correspond to the contributions to (2.34) due to $i=0$ and $i>0$, respectively. Applying (2.31) and (2.26), we get

$$
\begin{aligned}
\varphi_{l}^{(N)}(E) & \leq C^{N} \beta^{N}(l+1)^{-d / 2}+C_{1} C^{N} \beta^{N} \sum_{a=0}^{m-1}(l-a)^{-d / 2} \\
& \leq C_{1}^{N} \beta^{N} m(l-m+1)^{-d / 2} \quad(N \geq 1) .
\end{aligned}
$$

Combination of $(2.25),(2.33)$ and (2.36) completes the proof. The factor $\beta^{N}$ permits the sum over $N$ to be performed, for $\beta$ sufficiently small.

\section{Proof of Theorem 1.2}

The proof of Theorem 1.2 uses a lace expansion for $\mathbb{Q}_{n}(E)$ defined in (1.6). This expansion is again a modification of the Nguyen-Yang lace expansion for oriented percolation, but is different from the expansion of Section 2.1. We derive the modified expansion in Section 3.1, and use it to prove existence of the measure $\mathbb{Q}_{\infty}$ in Section 3.2. We will derive the same formula for $\mathbb{Q}_{\infty}(E)$ as was obtained in $(2.29)$ for $\mathbb{P}_{\infty}(E)$, thereby proving $\mathbb{Q}_{\infty}=\mathbb{P}_{\infty}$.

\subsection{The lace expansion for $\mathbb{Q}_{n}$}

Throughout this section, we fix $p \in\left[0,\|D\|_{\infty}^{-1}\right]$ and $m \geq 0$. Recall from (1.5), (1.6) and (1.8) that $S_{n}=\{(0,0) \longrightarrow n\}, \theta_{n}=\mathbb{P}\left(S_{n}\right)$, and $\mathbb{Q}_{n}(E)=\theta_{n}^{-1} \mathbb{P}\left(E \cap S_{n}\right)$. For $E \in \mathcal{E}_{m}, n \geq m$, and $0 \leq t \leq n$, we define

$$
\begin{aligned}
\theta_{n, t}(E) & =\mathbb{P}(E \cap\{(0,0) \longrightarrow n \text { with exactly } t \text { occupied pivotal bonds }\}), \\
\theta_{n}(E) & =\mathbb{P}(E \cap\{(0,0) \longrightarrow n\})=\sum_{t=0}^{n} \theta_{n, t}(E),
\end{aligned}
$$

where the pivotal bonds are pivotal for the event $S_{n}$. Then (1.6) reads

$$
\mathbb{Q}_{n}(E)=\frac{\theta_{n}(E)}{\theta_{n}}
$$

We will obtain formulas for $\theta_{n, t}(E)$ and $\mathbb{Q}_{n}(E)$ analogous to (2.7) and (2.19). We again regard the cluster of the origin in a configuration contributing to $\theta_{n}(E)$ as a string of sausages, but now the top sausage may be open at the top, as depicted in Figure 3. 


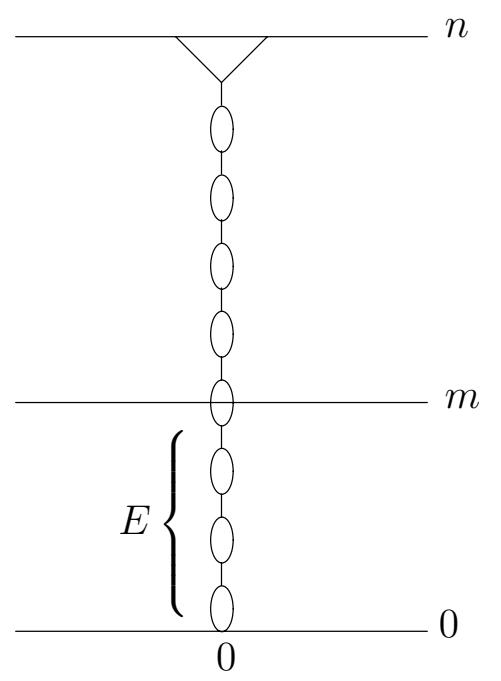

Figure 3: Schematic depiction of a configuration contributing to $\theta_{n}(E)$ as a string of sausages, with the top sausage open at the top. The event $E \in \mathcal{E}_{m}$ is required to occur.

Before beginning the expansion, with the help of Figures 1 and 3 we provide some intuition supporting the conjecture that $\mathbb{Q}_{n}, \mathbb{P}_{n}$ and $\mathbb{P}_{n}^{(x)}$ of (1.10) all converge to the same limiting measure, in arbitrary dimensions. The basic idea is that the number of pivotal bonds for the event $\{(0,0) \longrightarrow$ $(x, n)\}$ should diverge with $n$, so that the top sausage in Figure 1 begins near $n$, far beyond $m$. In the limit $n \rightarrow \infty$, the $x$-dependence inherent in locating the top of the top sausage in Figure 1 at $(x, n)$ should be of no importance for an event $E \in \mathcal{E}_{m}$ with $m$ fixed. Thus we expect the same limit whether $x$ is fixed as in $\mathbb{P}_{n}^{(x)}$ or summed over as in $\mathbb{P}_{n}$. Similarly, the number of pivotal bonds for the event $S_{n}$ should diverge with $n$, so that the top sausage in Figure 3 begins far above $m$. In the limit $n \rightarrow \infty$, the fact that the top sausage is open, rather than closed at some $(x, n)$, should be irrelevant for an event $E \in \mathcal{E}_{m}$. This supports the statement that $\mathbb{Q}_{\infty}=\mathbb{P}_{\infty}$.

To begin to set up the expansion, we let $(w, k) \Longrightarrow n$ denote the event that there exist $x, y \in \mathbb{Z}^{d}$ with bond-disjoint paths from $(w, k)$ to $(x, n)$ and from $(w, k)$ to $(y, n)$. Given $t>0$ and $\vec{b} \in B_{t}(n)$, we again set $\bar{b}_{0}=(0,0)$ and $\underline{b}_{t+1}=n$. We define

$$
U_{i j}^{\prime}(t)=U_{i j} \quad(0 \leq i<j \leq t-1), \quad U_{i t}^{\prime}(t)=-I\left[\bar{b}_{i} \Longrightarrow n\right] \quad(0 \leq i \leq t-1) .
$$

As in (2.6), (2.8) and (2.10), for $0 \leq i \leq j \leq t$ we define

$$
K_{t}^{\prime}[i, j]=\prod_{i \leq i^{\prime}<j^{\prime} \leq j}\left(1+U_{i^{\prime} j^{\prime}}^{\prime}(t)\right), \quad J_{t}^{\prime}[i, j]=\sum_{\Gamma \in \mathcal{G}[i, j]} \prod_{i^{\prime} j^{\prime} \in \Gamma} U_{i^{\prime} j^{\prime}}^{\prime}(t),
$$

and

$$
M_{t}^{\prime}[0, s ; m]=\sum_{i=0}^{s} K_{t}^{\prime}[0, i-1] J_{t}^{\prime}[i, s] I\left[\bar{b}_{i} \leq m \leq \underline{b}_{s+1}\right] .
$$

For $\vec{b} \in B_{t}(n)$, we define

$$
T_{t}(\vec{b}, n)= \begin{cases}\{(0,0) \Longrightarrow n\} & (t=0) \\ \bigcap_{i=1}^{t}\left\{b_{i} \text { occupied }\right\} \bigcap_{j=0}^{t-1}\left\{\bar{b}_{j} \Longrightarrow \underline{b}_{j+1}\right\} \cap\left\{\bar{b}_{t} \Longrightarrow n\right\} & (1 \leq t \leq n) .\end{cases}
$$


As in (2.7), (3.1) then becomes

$$
\theta_{n, t}(E)= \begin{cases}\mathbb{P}(E \cap\{(0,0) \Longrightarrow n\}) & (t=0) \\ \sum_{\vec{b} \in B_{t}(n)} \mathbb{E}\left[I[E] I\left[T_{t}(\vec{b}, n)\right] K_{t}^{\prime}[0, t]\right] & (1 \leq t \leq n) .\end{cases}
$$

For $0 \leq s \leq t$, we define

$$
\phi_{l, s}(E)= \begin{cases}\mathbb{P}(E \cap\{(0,0) \Longrightarrow n\}) & (s=0) \\ \sum_{\vec{b} \in B_{s}(l)} \mathbb{E}\left[I[E] I\left[T_{s}(\vec{b}, l)\right] M_{t}^{\prime}[0, s ; m]\right] & (1 \leq s \leq l) .\end{cases}
$$

It then follows exactly as in the proof of Lemma 2.1 that

$$
\theta_{n, t}(E)=\sum_{l=m}^{n-1} \sum_{s=0}^{t-1} \phi_{l, s}(E) p \theta_{n-l-1, t-s-1}+\phi_{n, t}(E) \quad(0 \leq t \leq n),
$$

where the first term on the right side is interpreted as zero when $t=0$. Since $U_{i j}^{\prime}(t)=U_{i j}$ when $0 \leq i<j<t$ by (3.4), it follows from (2.6), (2.8), (2.10)-(2.11), (3.5)-(3.6) and (3.9) that

$$
\phi_{l, s}(E)=\varphi_{l, s}(E) \quad(0 \leq s \leq t-1) .
$$

Therefore, $\phi_{l, s}$ in $(3.10)$ can be replaced with $\varphi_{l, s}$, except $\phi_{n, t}(E)$. Summation of (3.10) over $t=0, \ldots, n$, after this replacement, then gives

$$
\theta_{n}(E)=\sum_{l=m}^{n-1} \varphi_{l}(E) p \theta_{n-l-1}+\phi_{n}(E)
$$

with

$$
\phi_{n}(E)=\sum_{t=0}^{n} \phi_{n, t}(E)
$$

Combining (3.3) with (3.12), we get

$$
\mathbb{Q}_{n}(E)=\frac{1}{\theta_{n}}\left[\sum_{l=m}^{n-1} \varphi_{l}(E) p \theta_{n-l-1}+\phi_{n}(E)\right],
$$

which is analogous to (2.19).

Finally, we rewrite the expansion for $\phi_{n}(E)$ in terms of laces, as in (2.23)-(2.25). This yields

$$
\phi_{n}(E)=\sum_{N=0}^{\infty}(-1)^{N} \phi_{n}^{(N)}(E)
$$

with

$$
\begin{aligned}
\phi_{n}^{(0)}(E)= & \mathbb{P}_{p}(E \cap\{(0,0) \Longrightarrow n\}) \\
& +\sum_{t=1}^{n} \sum_{\vec{b} \in B_{t}(n)} \mathbb{E}\left(I[E] I\left[T_{t}(\vec{b}, n)\right] K_{t}[0, t-1] I\left[\bar{b}_{t} \leq m\right]\right), \\
\phi_{n}^{(N)}(E)= & \sum_{t=1}^{n} \sum_{\vec{b} \in B_{t}(n)} \mathbb{E}\left(I[E] I\left[T_{t}(\vec{b}, n)\right] \sum_{i=0}^{t-1} K_{t}[0, i-1] J_{t}^{(N)}[i, t] I\left[\bar{b}_{i} \leq m\right]\right) \quad(N \geq 1) .
\end{aligned}
$$

Here, $J_{t}^{(N)}[i, t]$ is obtained after replacing $U_{i j}$ by $U_{i j}^{\prime}(t)$ in (2.22). Equations (3.14)-(3.17), in combination with $(2.23)-(2.25)$, constitute the lace expansion for $\mathbb{Q}_{n}$. 


\subsection{Estimates on the lace expansion for $\mathbb{Q}_{n}$}

Throughout this section, we fix $p=p_{c}$. To prove Theorem 1.2, we will use (1.9), (3.14) and the following lemma.

Lemma 3.1. Let $d+1>4+1, p=p_{c}$ and $E \in \mathcal{E}_{m}$. Assume (1.9). There is an $L_{0}=L_{0}(d)$ such that for $L \geq L_{0}$,

$$
\lim _{n \rightarrow \infty} \frac{\phi_{n}(E)}{\theta_{n}}=0 .
$$

Proof of Theorem 1.2 subject to Lemma 3.1. Let $E \in \mathcal{E}_{m}$. By (3.14),

$$
\mathbb{Q}_{\infty}(E)=\lim _{n \rightarrow \infty} \mathbb{Q}_{n}(E)=\lim _{n \rightarrow \infty} \frac{1}{\theta_{n}}\left[\sum_{l=m}^{n-1} \varphi_{l}(E) p_{c} \theta_{n-l-1}+\phi_{n}(E)\right] .
$$

The second term vanishes in the limit, by Lemma 3.1. Given a small $a>0$, we decompose the first term as

$$
\sum_{l=m}^{\left\lfloor n^{1-a}\right\rfloor} \varphi_{l}(E) p_{c} \frac{\theta_{n-l-1}}{\theta_{n}}+\sum_{l=\left\lfloor n^{1-a}\right\rfloor+1}^{n-1} \varphi_{l}(E) p_{c} \frac{\theta_{n-l-1}}{\theta_{n}} .
$$

Using Lemma 2.2 to bound $\varphi_{l}(E)$ and (1.9) to bound the ratio of survival probabilities, we find that the second term in (3.20) is bounded above by

$$
K m \beta \sum_{l=\left\lfloor n^{1-a}\right\rfloor+1}^{n-1}(l-m+1)^{-d / 2} \mathcal{O}(n),
$$

which vanishes in the limit $n \rightarrow \infty$ when $d>4$ and $a$ is sufficiently small. Similarly, the first term in (3.20) can be analysed using Lemma 2.2, (1.9) and the dominated convergence theorem. This leads to the conclusion that

$$
\mathbb{Q}_{\infty}(E)=p_{c} \sum_{l=m}^{\infty} \varphi_{l}(E) .
$$

Comparing with the formula $(2.29)$ for $\mathbb{P}_{\infty}(E)$, we see that the limit defining $\mathbb{Q}_{\infty}(E)$ exists for every cylinder event $E$, and that it is equal to $\mathbb{P}_{\infty}(E)$. In view of Theorem 1.1 , this proves Theorem 1.2.

Proof of Lemma 3.1. The proof is somewhat technical. We start by bounding $\phi_{n}^{(0)}(E)$, defined in (3.16). In all our estimates, we will use $I[E] \leq 1$. The first term on the right side of (3.16) is bounded above by $\theta_{n}^{2}$, via the BK inequality. The second term on the right side of (3.16) is bounded above by

$$
\sum_{a=0}^{m-1} \sum_{u, v \in \mathbb{Z}^{d}} \mathbb{P}((0,0) \longrightarrow(u, a) \longrightarrow(v, a+1) \Longrightarrow n),
$$

where $((u, a),(u, a+1))$ represents the bond $b_{t}$. By the BK inequality, this is bounded above by $\sum_{a=0}^{m-1} \tau_{a} p_{c} \theta_{n-a-1}^{2}$, and therefore, using (2.26) and the monotonicity of $\theta_{n}$, we get

$$
\frac{\phi_{n}^{(0)}(E)}{\theta_{n}} \leq \theta_{n}+C m \frac{\theta_{n-m}^{2}}{\theta_{n}} .
$$


By (1.9), this goes to zero as $n \rightarrow \infty$.

Next we bound $\phi_{n}^{(N)}(E)$ for $N \geq 1$, defined in (3.17). For $N \geq 1$, let

$$
\Psi_{n}^{(N)}=\sum_{t=1}^{n} \sum_{\vec{b} \in B_{t}(m)} \mathbb{E}\left[I\left[T_{t}(\vec{b}, n)\right] J_{t}^{(N)}[0, t]\right]
$$

where, as explained under (3.17),

$$
J_{t}^{(N)}[0, t]=\sum_{L \in \mathcal{L}^{(N)}[0, t]} \prod_{i j \in L}\left(-U_{i j}^{\prime}(t)\right) \prod_{i^{\prime} j^{\prime} \in \mathcal{C}(L)}\left(1+U_{i^{\prime} j^{\prime}}^{\prime}(t)\right) .
$$

Using $I[E] \leq 1$ in $(3.17)$, and then factoring as in the proof of Lemma 2.1, we obtain the estimate

$$
\phi_{n}^{(N)}(E) \leq \Psi_{n}^{(N)}+\sum_{a=0}^{m-1} \tau_{a} p_{c} \Psi_{n-a-1}^{(N)} \leq \Psi_{n}^{(N)}+C \sum_{a=0}^{m-1} \Psi_{n-a-1}^{(N)} \leq C \sum_{a=0}^{m} \Psi_{n-a}^{(N)} .
$$

Consider first the case $N=1$. The unique lace in $\mathcal{L}^{(1)}[0, t]$ is $0 t$, and hence $J_{t}^{(1)}[0, t]$ contains a factor $-U_{0 t}(t)$, which implies that $0 \Longrightarrow n$. The event $T_{t}(\vec{b}, n)$ implies connections $(0,0) \Longrightarrow$ $\underline{b}_{1} \longrightarrow \bar{b}_{1} \longrightarrow n$. Moreover, the factor $1+U_{1 t}^{\prime}(t)$ in the product over $\mathcal{C}(L)$ in $J_{t}^{(1)}[0, t]$ implies that $\bar{b}_{1}$ is not doubly-connected to $n$. Thus $\Psi_{n}^{(1)}$ is bounded above by the probability of the disjoint connections depicted in Figure 4. Using the BK inequality, we therefore get

$$
\Psi_{n}^{(1)} \leq \sum_{j=0}^{n} \sum_{i=0}^{j} \sum_{x, y \in \mathbb{Z}^{d}} \tau_{j}(x) \tau_{i}(y) \tau_{j-i}(x-y) \theta_{n-j} \theta_{n-i} \leq \sum_{j=0}^{n} \theta_{n-j}^{2} \sum_{i=0}^{j} \sum_{x, y \in \mathbb{Z}^{d}} \tau_{j}(x) \tau_{i}(y) \tau_{j-i}(x-y)
$$

where we used the monotonicity of $\theta_{n}$ in the second inequality. The right side of (3.28) can be easily bounded from above by using the methods and results of [14]. In fact, since $\left\|\tau_{n}\right\|_{\infty} \leq K(n+1)^{-d / 2}$ by $[14$, Theorem $1.1(\mathrm{c})]$, it follows from (2.26) that

$$
\Psi_{n}^{(1)} \leq \sum_{j=0}^{n} \theta_{n-j}^{2} \sum_{i=0}^{j}\left\|\tau_{j}\right\|_{\infty} \tau_{i} \tau_{j-i} \leq C \sum_{j=0}^{n} \theta_{n-j}^{2}(j+1)^{-(d-2) / 2} .
$$

Using (1.9), we find that

$$
\Psi_{n}^{(1)} \leq C \sum_{j=0}^{n}(n-j+1)^{-2}(j+1)^{-(d-2) / 2} \leq C(n+1)^{-(2 \wedge(d-2) / 2)} .
$$

Therefore, (1.9) and (3.27) yield

$$
\frac{\phi_{n}^{(1)}(E)}{\theta_{n}} \leq C n \sum_{a=0}^{m}(n-a+1)^{-(2 \wedge(d-2) / 2)} \leq C m^{2}(n-m+1)^{-(1 \wedge(d-4) / 2)} .
$$

The right side goes to zero as $n \rightarrow \infty$, when $d>4$.

Before proceeding with $N \geq 2$, it is worth noting that the $\operatorname{sum} \sum_{i=0}^{j} \sum_{x, y \in \mathbb{Z}^{d}} \tau_{j}(x) \tau_{i}(y) \tau_{j-i}(x-y)$ in (3.28) can be bounded using another method from [14]. The above sum can be obtained from the simpler sum $\sum_{x \in \mathbb{Z}^{d}} \tau_{j}(x)^{2}$ by replacing one factor $\tau_{j}(x)$ by $\tau_{i}(y) \tau_{j-i}(x-y)$ and then summing 


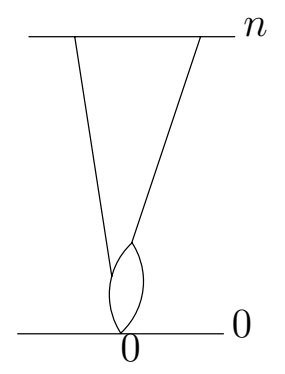

Figure 4: Schematic depiction of disjoint connections required by $\Psi_{n}^{(1)}$.

over $y$ and $i$. The first part of this procedure is referred to in [14, Definition 4.1] as Construction $1^{\lambda}(y, i)$, where $\lambda$ labels the diagram line that is modified. According to [14, Lemma 4.6(a)], the diagram obtained after Construction $1^{\lambda}(y, i)$ followed by summation over $y$ obeys the same bound as the original diagram, up to a multiplicative constant. Thus, Construction $1^{\lambda}(y, i)$ followed by summation over $y$ produces a diagram that is bounded by a constant multiple of the bound on $\sum_{x \in \mathbb{Z}^{d}} \Pi_{j}^{(0)}(x)$, namely the bound $C(j+1)^{-d / 2}$ of (2.31) (we have omitted the factor $\beta$ from (2.31) to allow for the possibility that $j=0$ ). The bound (3.29) could thus be replaced by

$$
\Psi_{n}^{(1)} \leq \sum_{j=0}^{n} \theta_{n-j}^{2} \sum_{i=0}^{j} C(j+1)^{-d / 2} \leq C \sum_{j=0}^{n} \theta_{n-j}^{2}(j+1)^{-(d-2) / 2},
$$

which yields the same conclusion as (3.29). In dealing with $N \geq 2$, we will prefer the above method using Construction $1^{\lambda}(y, i)$, rather than the method of the previous paragraph. In (3.32), we have bounded $\Psi_{n}^{(1)}$ using $\Pi_{j}^{(0)}$. Similarly, for $N \geq 2$, we will bound $\Psi_{n}^{(N)}$ using $\Pi_{j}^{(N-1)}$ with $0 \leq j \leq n$.

Fix $N \geq 2$. We begin with a decomposition of $J_{t}^{(N)}[0, t]$. We write a lace $L \in \mathcal{L}^{(N)}[0, t]$ in the form $L=\left\{i_{1} j_{1}, \ldots, i_{N} j_{N}\right\}$, with $0=i_{1}<i_{2}<\cdots<i_{N}<t$. We write $L$ as $L^{-} \cup\left\{i_{N} t\right\}$, where $L^{-} \in \mathcal{L}^{(N-1)}\left[0, j_{N-1}\right]$. Let $\ell\left(L^{-}\right)=1$ if $N=2$, and $\ell\left(L^{-}\right)=j_{N-2}$ if $N \geq 3$. Then we may write

$$
\sum_{L \in \mathcal{L}^{(N)}[0, t]}=\sum_{j_{N-1}=1}^{t-1} \sum_{L^{-} \in \mathcal{L}^{(N-1)}\left[0, j_{N-1}\right]} \sum_{i_{N}=\ell\left(L^{-}\right)}^{j_{N-1}-1} .
$$

We make the decomposition

$$
\prod_{i j \in L}\left(-U_{i j}^{\prime}(t)\right)=\left(-U_{i_{N} t}^{\prime}(t)\right) \prod_{i j \in L^{-}}\left(-U_{i j}\right)
$$

and note that

$$
\mathcal{C}\left(L^{-}\right) \cup\left\{i_{N} r: i_{N}<r<t\right\} \subset \mathcal{C}(L) .
$$

Therefore

$$
\begin{aligned}
J_{t}^{(N)}[0, t] \leq & \sum_{j_{N-1}=1}^{t-1} \sum_{L^{-} \in \mathcal{L}^{(N-1)}\left[0, j_{N-1}\right]} \prod_{i j \in L^{-}}\left(-U_{i j}\right) \prod_{i^{\prime} j^{\prime} \in \mathcal{C}\left(L^{-}\right)}\left(1+U_{i^{\prime} j^{\prime}}\right) \\
& \times \sum_{i_{N}=\ell\left(L^{-}\right)}^{j_{N-1}-1}\left(-U_{i_{N} t}^{\prime}(t)\right) \prod_{i_{N}<r<t}\left(1+U_{i_{N} r}\right) .
\end{aligned}
$$




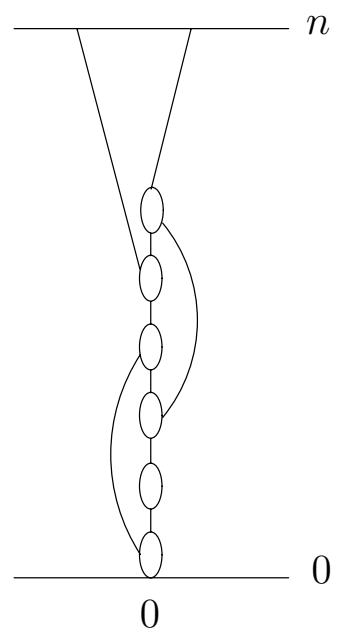

Figure 5: Example of disjoint connections required by a configuration contributing to $\Psi_{n}^{(3)}$.

Were it not for the dependence of the second line of (3.36) on $L^{-}$through the lower limit of summation over $i_{N}$, we would be able to rewrite the sum over $L^{-}$in the first line simply as $J^{(N-1)}\left[0, j_{N-1}\right]$. The effect of the second line is twofold. First, the factor $\left(-U_{i_{N} t}^{\prime}(t)\right)$ ensures that $\bar{b}_{i_{N}} \Longrightarrow n$. Second, together with the indicator $I\left[T_{t}(\vec{b}, n)\right]$, the factor $\prod_{i_{N}<r<t}\left(1+U_{i_{N} r}\right)$ ensures that, in addition to the disjoint connections implied by $J^{(N-1)}\left[0, j_{N-1}\right]$ (leading to an upper bound by a diagram $\Pi^{(N-1)}$ ), there are additional disjoint connections $\underline{b}_{t} \longrightarrow n$ and $\bar{b}_{i_{N}} \longrightarrow n$ that accomplish the requirement that $\bar{b}_{i_{N}} \Longrightarrow n$. The required disjoint connections are depicted schematically in Figure 5. These connections are the connections relevant for $\Pi^{(N-1)}$, together with a line from the top of the diagram representing $\Pi^{(N-1)}$ to $n$ and a line from a new vertex on $\Pi^{(N-1)}$ to $n$. Explicitly, we have the upper bound

$$
\Psi_{n}^{(N)} \leq \sum_{j=0}^{n} \sum_{i=0}^{j} \sum_{x, y \in \mathbb{Z}^{d}} \bar{\Pi}_{j}^{(N-1)}(x ;(y, i)) \theta_{n-i} \theta_{n-j}
$$

where $\bar{\Pi}_{j}^{(N-1)}(x ;(y, i))$ denotes the result of applying Construction $1^{\lambda}(y, i)$ to a diagram bounding $\Pi_{j}^{(N-1)}(x)$, followed by an appropriate sum over $\lambda$. By [14, Lemma 4.6(a)], $\sum_{x, y \in \mathbb{Z}^{d}} \bar{\Pi}_{j}^{(N-1)}(x ;(y, i))$ obeys the bound on $\sum_{x \in \mathbb{Z}^{d}} \Pi_{j}^{(N-1)}(x)$ of $(2.31)$, with a different constant. Since $\theta_{n-i} \leq \theta_{n-j}$, it follows that

$$
\Psi_{n}^{(N)} \leq \sum_{j=0}^{n} \sum_{i=0}^{j}(C \beta)^{N-1}(j+1)^{-d / 2} \theta_{n-j}^{2} .
$$

Via (1.9), this gives

$$
\Psi_{n}^{(N)} \leq\left(C^{\prime} \beta\right)^{N-1} \sum_{j=0}^{n}(j+1)^{-(d-2) / 2}(n-j+1)^{-2} \leq\left(C^{\prime} \beta\right)^{N-1}(j+1)^{-(2 \wedge(d-2) / 2)},
$$

and the desired result follows from (3.27) as in (3.31), again using (1.9). The factor $\beta^{N-1}$ permits the summation over $N$ to be performed, for $\beta$ sufficiently small. 


\section{Oriented percolation and super-Brownian motion}

\subsection{Convergence of moment measures}

The oriented percolation $r$-point functions are defined, for $n_{i} \geq 0$ and $x_{i} \in \mathbb{Z}^{d}$, by

$$
\tau_{n_{1}, \ldots, n_{r-1}}^{(r)}\left(x_{1}, \ldots, x_{r-1}\right)=\mathbb{P}_{p}\left((0,0) \longrightarrow\left(x_{i}, n_{i}\right) \text { for each } i=1, \ldots, r-1\right) .
$$

In particular, $\tau_{n}^{(2)}(x)$ is the two-point function $\tau_{n}(x)$. Given $m \in \mathbb{N}$, an absolutely summable function $f: \mathbb{Z}^{m d} \rightarrow \mathbb{C}$, and $\vec{k}=\left(k_{1}, \ldots, k_{m}\right)$ with each $k_{j} \in(-\pi, \pi]^{d}$, we define the Fourier transform

$$
\hat{f}(\vec{k})=\sum_{y_{1}, \ldots, y_{m} \in \mathbb{Z}^{d}} f(\vec{y}) e^{i \vec{k} \cdot \vec{y}}
$$

where $\vec{k} \cdot \vec{y}=\sum_{j=1}^{m} k_{j} \cdot y_{j}$. When $m=1$, we write simply $k$ in place of $\vec{k}$.

In [14], the Fourier transforms of (4.1) are related, in an appropriate scaling limit, to the Fourier transforms of the moment measures of the canonical measure of super-Brownian motion $[18,22]$. The canonical measure of super-Brownian motion is a certain scaling limit of critical branching random walk, started from a single particle located at the origin. It is a Markov process whose state $X_{t}$ at time $t>0$ is a finite non-negative measure on $\mathbb{R}^{d}$. By definition, its $l^{\text {th }}$ moment measure has Fourier transform

$$
\hat{M}_{\vec{t}}^{(l)}(\vec{k})=E\left(\int_{\mathbb{R}^{d l}} X_{t_{1}}\left(d x_{1}\right) \cdots X_{t_{l}}\left(d x_{l}\right) \prod_{j=1}^{l} e^{i k_{j} x_{j}}\right),
$$

where $\vec{t}=\left(t_{1}, \ldots, t_{l}\right)$ with each $t_{i} \in(0, \infty)$, and $\vec{k}=\left(k_{1}, \ldots, k_{l}\right)$ with each $k_{i} \in \mathbb{R}^{d}$.

The following result is a combination of [14, Theorems 1.1(a) and 1.2] with [14, (1.25)]. In its statement, the parameter $\epsilon$ is fixed such that $\sum_{x \in \mathbb{Z}^{d}}|x|^{2+2 \epsilon} D(x) \leq C L^{2+2 \epsilon}$. The existence of such an $\epsilon>0$ is part of the assumptions on $D$ from [14] discussed in Section 1.2 and assumed in this paper.

Theorem 4.1. Let $d>4, p=p_{c}, \delta \in\left(0,1 \wedge \epsilon \wedge \frac{d-4}{2}\right), r \geq 2, \vec{t}=\left(t_{1}, \ldots, t_{r-1}\right) \in(0, \infty)^{r-1}$, and $\vec{k}=\left(k_{1}, \ldots, k_{r-1}\right) \in \mathbb{R}^{(r-1) d}$. There exist $L_{0}=L_{0}(d)$ and finite positive constants $A=A(d, L)$, $v=v(d, L), V=V(d, L)$ (with $L_{0}, A, v, V$ independent of $r$ ) such that for $L \geq L_{0}$,

$$
\hat{\tau}_{\lfloor n \vec{t}\rfloor}^{(r)}\left(\vec{k} / \sqrt{v \sigma^{2} n}\right)=A^{2 r-3} V^{r-2} n^{r-2}\left[\hat{M}_{\vec{t}}^{(r-1)}(\vec{k})+\mathcal{O}\left(n^{-\delta}\right)\right] .
$$

Rather than applying Theorem 4.1 directly, we use an auxiliary result that was derived in [14] in the course of proving Theorem 4.1. Let $\bar{n}$ denote the second largest component of $\vec{n}=$ $\left(n_{1}, \ldots, n_{r-1}\right)$. In Section 5, we will use [14, (2.52)], which states that

$$
\hat{\tau}_{\vec{n}}^{(r)}\left(\vec{k} / \sqrt{v \sigma^{2} n}\right)=A\left(A^{2} V\right)^{r-2} n^{r-2}\left[\hat{M}_{\vec{n} / n}^{(r-1)}(\vec{k})+\mathcal{O}\left((\bar{n}+1)^{-\delta}\right)\right] \quad(r \geq 3)
$$

holds uniformly in $n \geq \bar{n}$. 


\subsection{The moment measures of super-Brownian motion}

In Section 5, we will make use of elementary properties of the $\hat{M}_{\vec{t}}^{(l)}(\vec{k})$, which we now summarise. For $l=1$,

$$
\hat{M}_{t}^{(1)}(\vec{k})=e^{-|k|^{2} t / 2 d} .
$$

For $l>1$, the $\hat{M}_{\vec{t}}^{(l)}(\vec{k})$ are given recursively by

$$
\hat{M}_{\vec{t}}^{(l)}(\vec{k})=\int_{0}^{\underline{t}} d t \hat{M}_{t}^{(1)}\left(k_{1}+\cdots+k_{l}\right) \sum_{I \subset J_{1}:|I| \geq 1} \hat{M}_{\vec{t}_{I}-t}^{(i)}\left(\vec{k}_{I}\right) \hat{M}_{\vec{t}_{J \backslash I}-t}^{(l-i)}\left(\vec{k}_{J \backslash I}\right),
$$

where $i=|I|, J=\{1, \ldots, l\}, J_{1}=J \backslash\{1\}, \underline{t}=\min _{i} t_{i}, \vec{t}_{I}$ denotes the vector consisting of the components $t_{i}$ of $\vec{t}$ with $i \in I$, and $\vec{t}_{I}-t$ denotes subtraction of $t$ from each component of $\vec{t}_{I}$ [9]. The explicit solution to the recursive formula (4.7) can be found in $[14,(1.25)]$. For example,

$$
\hat{M}_{t_{1}, t_{2}}^{(2)}\left(k_{1}, k_{2}\right)=\int_{0}^{t_{1} \wedge t_{2}} d t e^{-\left|k_{1}+k_{2}\right|^{2} t / 2 d} e^{-\left|k_{1}\right|^{2}\left(t_{1}-t\right) / 2 d} e^{-\left|k_{2}\right|^{2}\left(t_{2}-t\right) / 2 d} .
$$

Equation (4.8) is a statement, in Fourier language, that mass arrives at given points $\left(x_{1}, t_{1}\right),\left(x_{2}, t_{2}\right)$ via a Brownian path from the origin that splits into two Brownian paths at a time chosen uniformly from the interval $\left[0, t_{1} \wedge t_{2}\right]$. The recursive formula (4.7) has a related interpretation for all $l \geq 2$, in which $t$ is the time of the first branching. The sets $I$ and $J \backslash I$ label the offspring of each of the two particles after the first branching.

Lemma 4.2. (a) For $k \in \mathbb{R}^{d}$,

$$
\hat{M}_{1,1}^{(2)}(0, k)=e^{-\frac{|k|^{2}}{2 d}}
$$

(b) For $l \geq 0, t \geq s$ and $k_{j} \in \mathbb{R}^{d}$,

$$
\hat{M}_{t, s, \ldots, s}^{(l+1)}\left(0, k_{2}, \ldots, k_{l}\right)=\hat{M}_{s, s, \ldots, s}^{(l+1)}\left(0, k_{2}, \ldots, k_{l}\right) .
$$

(c) For $l \geq 0$,

$$
\hat{M}_{t, \ldots, t}^{(l+1)}(\overrightarrow{0})=t^{l} 2^{-l}(l+1) !
$$

Proof. (a) This follows immediately from (4.8).

(b) The proof is by induction on $l$. For $l=0$, both sides of (4.10) equal 1 , by (4.6). For $l \geq 1$, we use (4.7) with $\vec{t}=(t, s, \ldots, s)$ to obtain

$$
\hat{M}_{t, s, \ldots, s}^{(l+1)}\left(0, k_{2}, \ldots, k_{l}\right)=\int_{0}^{s} d u \hat{M}_{u}^{(1)}\left(k_{2}+\cdots+k_{l}\right) \sum_{I \subset J_{1}:|I| \geq 1} \hat{M}_{\vec{t}_{I}-u}^{(i)}\left(\vec{k}_{I}\right) \hat{M}_{\vec{t}_{J \backslash I}-u}^{(l-i)}\left(\vec{k}_{J \backslash I}\right) .
$$

On the right side, all the arguments in $\vec{t}_{I}-u$ and $\vec{t}_{J \backslash I}-u$ are equal to $s-u$, except for one, which is $t-u$. The distinguished time variable also has $k_{1}=0$. Applying the induction hypothesis, we get

$$
\begin{aligned}
\hat{M}_{t, s, \ldots, s}^{(l+1)}\left(0, k_{2}, \ldots, k_{l}\right) & =\int_{0}^{s} d u \hat{M}_{u}^{(1)}\left(k_{2}+\cdots+k_{l}\right) \sum_{I \subset J_{1}:|I| \geq 1} \hat{M}_{s-u, \ldots, s-u}^{(i)}\left(\vec{k}_{I}\right) \hat{M}_{s-u, \ldots, s-u}^{(l-i+1)}\left(\vec{k}_{J \backslash I}\right) \\
& =\hat{M}_{s, s, \ldots, s}^{(l+1)}\left(0, k_{2}, \ldots, k_{l}\right)
\end{aligned}
$$


which advances the induction and proves (4.10).

(c) The proof is again by induction on $l$. For $l=0$, (4.11) follows from (4.6). For $l \geq 1$ we use (4.7) and the induction hypothesis to obtain

$$
\begin{aligned}
\hat{M}_{\vec{t}}^{(l+1)}(\overrightarrow{0}) & =\int_{0}^{t} d s \sum_{i=1}^{l}\left(\begin{array}{l}
l \\
i
\end{array}\right)(t-s)^{i-1} 2^{-(i-1)} i !(t-s)^{l-i} 2^{-(l-i)}(l-i+1) ! \\
& =2^{-(l-1)} \sum_{i=1}^{l}\left(\begin{array}{l}
l \\
i
\end{array}\right) i !(l-i+1) ! \int_{0}^{t}(t-s)^{l-1} d s \\
& =t^{l} 2^{-(l-1)}(l-1) ! \sum_{i=1}^{l}(l-i+1)=t^{l} 2^{-l}(l+1) !,
\end{aligned}
$$

which advances the induction and proves (4.11).

\section{Proof of Theorems 1.3-1.5}

\subsection{Proof of Theorem 1.3}

Before proving Theorem 1.3, we first derive upper and lower bounds on the IIC two-point function, defined by

$$
\rho_{m}(y)=\mathbb{P}_{\infty}((0,0) \longrightarrow(y, m))=\lim _{n \rightarrow \infty} \frac{1}{\tau_{n}} \sum_{x \in \mathbb{Z}^{d}} \tau_{n, m}^{(3)}(x, y) .
$$

In addition to the fact that $\tau_{n} \rightarrow A$ by (2.26), we will use the fact that

$$
\sup _{x \in \mathbb{Z}^{d}} \tau_{n}(x) \leq C(n+1)^{-d / 2}
$$

by $[14$, Theorem $1.1(\mathrm{c})]$.

Beginning with the upper bounds, we show that

$$
\sum_{y \in \mathbb{Z}^{d}} \rho_{m}(y) \leq C m, \quad \sup _{y \in \mathbb{Z}^{d}} \rho_{m}(y) \leq C(m+1)^{-(d-2) / 2} .
$$

For the first bound in (5.3), we use the tree-graph bound [2] to obtain the estimate

$$
\tau_{n, m}^{(3)}(x, y) \leq \sum_{z \in \mathbb{Z}^{d}} \sum_{l=0}^{m} \tau_{l}(z) \tau_{m-l}(y-z) \tau_{n-l}(x-z) .
$$

Therefore, by (5.1) and (2.26),

$$
\rho_{m}(y) \leq C \sum_{z \in \mathbb{Z}^{d}} \sum_{l=0}^{m} \tau_{l}(z) \tau_{m-l}(y-z) .
$$

Summing over $y$ and again using (2.26), we get the first bound of (5.3). For the second bound in (5.3), we apply (5.2) to either the first or the second factor on the right side of (5.5), according to whether $l \geq m / 2$ or $l \leq m / 2$. This gives, as required,

$$
\sup _{y \in \mathbb{Z}^{d}} \rho_{m}(y) \leq C \sum_{l=0}^{m}(l \vee(m-l))^{-d / 2} \leq C(m+1)^{-(d-2) / 2} .
$$


Continuing with the lower bounds, we show that there is a constant $c>0$ such that

$$
\sum_{|y| \leq \sqrt{m}} \rho_{m}(y) \geq c m
$$

To prove this, we note that, by (5.1),

$$
\hat{\rho}_{m}(k)=\lim _{n \rightarrow \infty} \frac{1}{\tau_{n}} \hat{\tau}_{n, m}^{(3)}(0, k) .
$$

We use (4.5) with $\left(n_{1}, n_{2}\right)=(n, m), \bar{n}=m$, and with $n$ of (4.5) equal to $m$. Combining this with Lemma $4.2(\mathrm{a}, \mathrm{b})$, we get

$$
\lim _{n \rightarrow \infty} \hat{\tau}_{n, m}^{(3)}\left(0, \frac{k}{\sqrt{v \sigma^{2} m}}\right)=A\left(A^{2} V\right) m \hat{M}_{\frac{n}{m}, 1}^{(2)},(0, k)\left[1+\mathcal{O}\left(m^{-\delta}\right)\right]=A\left(A^{2} V\right) m e^{-\frac{|k|^{2}}{2 d}}\left[1+\mathcal{O}\left(m^{-\delta}\right)\right] .
$$

Therefore, using (5.8) and (2.26), we obtain

$$
\lim _{m \rightarrow \infty} \frac{1}{m A^{2} V} \hat{\rho}_{m}\left(k / \sqrt{v \sigma^{2} m}\right)=e^{-\frac{|k|^{2}}{2 d}},
$$

and hence the discrete measure on $\mathbb{R}^{d}$ that assigns mass $\left(m A^{2} V\right)^{-1} \rho_{m}(x)$ to $x / \sqrt{v \sigma^{2} m}\left(x \in \mathbb{Z}^{d}\right)$ converges weakly to a Gaussian. This implies (5.7).

Proof of Theorem 1.3. For the upper bound on $D_{R}$, we use the decomposition

$$
D_{R}=\sum_{m} \sum_{|y| \leq R} \rho_{m}(y)=\sum_{m \leq R^{2}} \sum_{|y| \leq R} \rho_{m}(y)+\sum_{m>R^{2}} \sum_{|y| \leq R} \rho_{m}(y) .
$$

By the first bound of (5.3), the first term is bounded above by $C \sum_{m \leq R^{2}} m=\mathcal{O}\left(R^{4}\right)$. By the second bound of (5.3), the second term is bounded above by

$$
\sum_{m>R^{2}} C R^{d} \sup _{y \in \mathbb{Z}^{d}} \rho_{m}(y) \leq C R^{d} \sum_{m>R^{2}}(m+1)^{-(d-2) / 2}=\mathcal{O}\left(R^{4}\right) .
$$

This proves the upper bound on $D_{R}$.

For the lower bound on $D_{R}$, we use that (5.7) implies

$$
D_{R} \geq \sum_{m \leq R^{2}} \sum_{|y| \leq R} \rho_{m}(y) \geq \sum_{m \leq R^{2}} \sum_{|y| \leq \sqrt{m}} \rho_{m}(y) \geq \sum_{m \leq R^{2}} c m \geq \frac{1}{2} c R^{4} .
$$

Finally, we make an observation about the scaling of the IIC $r$-point functions for general $r$, although we will not need this. Let $\vec{y}=\left(y_{1}, \ldots, y_{r-1}\right)$ and $\vec{m}=\left(m_{1}, \ldots, m_{r-1}\right)$ with $y_{i} \in \mathbb{Z}^{d}$, $m_{i} \in \mathbb{Z}_{+}$, and define the IIC $r$-point function by

$$
\rho_{\vec{m}}^{(r)}(\vec{y})=\mathbb{P}_{\infty}\left((0,0) \longrightarrow\left(y_{i}, m_{i}\right) \text { for all } i=1, \ldots, r-1\right) .
$$

In particular, $\rho_{m}^{(2)}(y)$ is the same as $\rho_{m}(y)$ of (5.1). The methods employed to prove (5.10) can also be used to show that

$$
\lim _{m \rightarrow \infty} \frac{1}{\left(m A^{2} V\right)^{r-1}} \hat{\rho}_{m \vec{t}}^{(r)}\left(\vec{k} / \sqrt{v \sigma^{2} m}\right)=\hat{M}_{1, \vec{t}}^{(r)}(0, \vec{k}),
$$

for all $r \geq 2, \vec{t}=\left(t_{1}, \ldots, t_{r-1}\right) \in(0,1]^{r-1}$ and $\vec{k} \in \mathbb{R}^{d(r-1)}$. 


\subsection{Proof of Theorem 1.4}

We first prove (1.16). Let $l \geq 1$. By (1.3), (1.14) and (4.1), we have

$$
\begin{aligned}
\mathbb{E}_{\mathbb{P}_{n}}\left[N_{m}^{l}\right] & =\frac{1}{\tau_{n}} \sum_{x \in \mathbb{Z}^{d}} \sum_{y_{1}, \ldots, y_{l} \in \mathbb{Z}^{d}} \mathbb{P}\left((0,0) \longrightarrow(x, n),(0,0) \longrightarrow\left(y_{i}, m\right) \text { for each } i=1, \ldots, l\right) \\
& =\frac{1}{\tau_{n}} \hat{\tau}_{n, m, \ldots, m}^{(l+2)}(\overrightarrow{0})
\end{aligned}
$$

We take $n \geq m$, and use (4.5) with $r=l+2, \vec{k}=\overrightarrow{0}, \vec{n}=(n, m, \ldots, m)$, and with $n$ of (4.5) equal to $\bar{n}=m$. This gives

$$
\hat{\tau}_{n, m, \ldots, m}^{(l+2)}(\overrightarrow{0})=A\left(A^{2} V\right)^{l} m^{l}\left[\hat{M}_{\frac{n}{m}, 1, \ldots, 1}^{(l+1)}(\overrightarrow{0})+\mathcal{O}\left(m^{-\delta}\right)\right] .
$$

Applying Lemma 4.2(b,c), we get

$$
\hat{\tau}_{n, m, \ldots, m}^{(l+2)}(\overrightarrow{0})=A\left(A^{2} V\right)^{l} m^{l}\left[2^{-l}(l+1) !+\mathcal{O}\left(m^{-\delta}\right)\right] .
$$

Combining (5.16) and (5.18), we find

$$
\mathbb{E}_{\mathbb{P}_{n}}\left[\left(\frac{N_{m}}{m}\right)^{l}\right]=\frac{A}{\tau_{n}}\left(A^{2} V\right)^{l}\left[2^{-l}(l+1) !+\mathcal{O}\left(m^{-\delta}\right)\right] .
$$

Taking the limit $n \rightarrow \infty$, and using (2.26), we arrive at

$$
\mathbb{E}_{\infty}\left[\left(\frac{N_{m}}{m}\right)^{l}\right]=\left(A^{2} V\right)^{l} 2^{-l}(l+1) !+\mathcal{O}\left(m^{-\delta}\right)
$$

and hence at (1.16) after letting $m \rightarrow \infty$.

The distribution of the size-biased exponential random variable is determined by its moments, since its moment generating function has a positive radius of convergence. It therefore follows from the convergence of moments expressed by (1.16) that $m^{-1} N_{m}$ converges weakly to a size-biased exponential random variable with parameter $\lambda=2 /\left(A^{2} V\right)$ (see [4, Theorem 30.2]). This completes the proof of Theorem 1.4.

\subsection{Proof of Theorem $\mathbf{1 . 5}$}

It follows from (1.6), (1.8), (1.14) and (4.1) that

$$
\mathbb{E}_{\mathbb{Q}_{m}}\left[N_{m}^{l}\right]=\frac{1}{\theta_{m}} \hat{\tau}_{\vec{m}}^{(l+1)}(\overrightarrow{0}),
$$

with $\vec{m}=(m, \ldots, m)$. As in the proof of Theorem 1.4 we find, now also with the help of (1.9), that

$$
\lim _{m \rightarrow \infty} \mathbb{E}_{\mathbb{Q}_{m}}\left[\left(\frac{N_{m}}{m}\right)^{l}\right]=\frac{2 B}{A V}\left(A^{2} V\right)^{l} 2^{-l} l ! \quad(l=1,2, \ldots) .
$$

Let $\alpha=2 B /(A V)$ and suppose for the moment that $\alpha=1$. Then (1.18) holds, and the right side of (5.22) gives the moments of an exponential random variable with parameter $\lambda=2 /\left(A^{2} V\right)$. It 
then follows as in the proof of Theorem 1.4 that $m^{-1} N_{m}$ converges to this exponential random variable in distribution. To complete the proof, it suffices to show that $\alpha=1$. We first prove that $\alpha \leq 1$ and then prove that $\alpha \geq 1$.

Proof that $\alpha \leq 1$. Since $\tau_{m}^{-1} N_{m}$ has expectation 1 under $\mathbb{P}$, we can define a new expectation by

$$
\mathbb{E}_{m}^{\prime}[X]=\mathbb{E}\left[\tau_{m}^{-1} N_{m} X\right]
$$

By definition of $\mathbb{E}_{m}^{\prime},(1.3)$ and $(5.16)$,

$$
\mathbb{E}_{m}^{\prime}\left[\left(\frac{N_{m}}{m}\right)^{l}\right]=\mathbb{E}_{\mathbb{P}_{m}}\left[\left(\frac{N_{m}}{m}\right)^{l}\right]=\frac{1}{m^{l} \tau_{m}} \hat{\tau}_{m, m, \ldots, m}^{(l+2)}(\overrightarrow{0})
$$

As in (5.20), it follows that the moments of $m^{-1} N_{m}$ under $\mathbb{E}_{m}^{\prime}$ converge to those of a size-biased exponential random variable with parameter $\lambda=2 /\left(A^{2} V\right)$. Therefore, under this measure, $m^{-1} N_{m}$ converges weakly to a size-biased exponential random variable. In particular, for real $t$, the moment generating function $\mathbb{E}_{m}^{\prime}\left[e^{-t \frac{N_{m}}{m}}\right]$ converges to that of a size-biased exponential distribution with parameter $\lambda$, which is $\frac{\lambda^{2}}{(\lambda+t)^{2}}$.

Let $t \geq 0$. In terms of $\mathbb{E}_{m}^{\prime}$, we can rewrite the moment generating function of $m^{-1} N_{m}$, under $\mathbb{Q}_{m}$, as (recall (1.5)-(1.6) and (1.8))

$$
\mathbb{E}_{\mathbb{Q}_{m}}\left[e^{-t \frac{N_{m}}{m}}\right]=1-\frac{1}{m} \mathbb{E}_{\mathbb{Q}_{m}}\left[\int_{0}^{t} N_{m} e^{-s \frac{N_{m}}{m}} d s\right]=1-\frac{\tau_{m}}{m \theta_{m}} \int_{0}^{t} \mathbb{E}_{m}^{\prime}\left[e^{-s \frac{N_{m}}{m}}\right] d s .
$$

By the dominated convergence theorem, together with (1.9) and (2.26), it follows from the identity $\alpha=A B \lambda$ that

$$
0 \leq \lim _{m \rightarrow \infty} \mathbb{E}_{\mathbb{Q}_{m}}\left[e^{-t \frac{N_{m}}{m}}\right]=1-A B \int_{0}^{t} \frac{\lambda^{2}}{(\lambda+s)^{2}} d s=1-\alpha+\alpha \frac{\lambda}{\lambda+t}
$$

By letting $t \rightarrow \infty$, we conclude from (5.26) that $\alpha \leq 1$.

Proof that $\alpha \geq 1$. Fix $s>0$. By definition (recall (1.5)-(1.6)),

$$
\theta_{\lfloor m(1+s)\rfloor}=\theta_{m} \mathbb{P}\left(S_{\lfloor m(1+s)\rfloor} \mid S_{m}\right) .
$$

Let $n$ be any positive integer and let $A \subset \mathbb{Z}^{d}$ be any finite set, and define

$$
\theta_{n}(A)=\mathbb{P}(\exists a \in A:(a, 0) \longrightarrow n)=1-\mathbb{P}(\forall a \in A:(a, 0) \nrightarrow n) .
$$

Since, for any $a \in \mathbb{Z}^{d},\{(a, 0) \nrightarrow n\}$ is a decreasing event, it follows from the FKG inequality that

$$
\theta_{n}(A) \leq 1-\left(1-\theta_{n}\right)^{|A|}
$$

Therefore, using $n=\lfloor m s\rfloor$ and $A=\left\{a \in \mathbb{Z}^{d}:(0,0) \longrightarrow(a, m)\right\}$, we have

$$
\theta_{\lfloor m(1+s)\rfloor} \leq \theta_{m} \mathbb{E}\left(1-\left(1-\theta_{\lfloor m s\rfloor}\right)^{N_{m}} \mid S_{m}\right)=\theta_{m}\left\{1-\mathbb{E}_{\mathbb{Q}_{m}}\left(\left(1-\theta_{\lfloor m s\rfloor}\right)^{N_{m}}\right)\right\},
$$


and hence, by (1.9), for any $\eta>0$ we have

$$
\begin{aligned}
\frac{1}{B(1+s)}=\lim _{m \rightarrow \infty} m \theta_{\lfloor m(1+s)\rfloor} & \leq \frac{1}{B}\left\{1-\lim _{m \rightarrow \infty} \mathbb{E}_{\mathbb{Q}_{m}}\left(\left(1-\theta_{\lfloor m s\rfloor}\right)^{m \frac{N_{m}}{m}}\right)\right\} \\
& \leq \frac{1}{B}\left\{1-\lim _{m \rightarrow \infty} \mathbb{E}_{\mathbb{Q}_{m}}\left(e^{-\left(\frac{1}{B s}+\eta\right) \frac{N_{m}}{m}}\right)\right\}
\end{aligned}
$$

With minor changes, the calculations leading to (5.26) can also be carried out for $t=-i u$ with $u \in \mathbb{R}$. This yields

$$
\lim _{m \rightarrow \infty} \mathbb{E}_{\mathbb{Q}_{m}}\left[e^{i u \frac{N m}{m}}\right]=1-\alpha+\alpha \frac{\lambda}{\lambda-i u} .
$$

It follows from (5.32) that $m^{-1} N_{m}$ under $\mathbb{Q}_{m}$ converges in distribution to a random variable $Y$ having the property that $\mathbb{P}(Y=0)=1-\alpha$ and that the distribution of $Y$ conditional on $Y>0$ is that of an exponential random variable with parameter $\lambda$. By (5.31), it follows that

$$
\frac{1}{B(1+s)} \leq \frac{1}{B}\left\{1-\mathbb{E}\left[e^{-\left(\frac{1}{B s}+\eta\right) Y}\right]\right\}=\frac{\alpha}{B}\left\{1-\mathbb{E}\left[e^{-\left(\frac{1}{B s}+\eta\right) Y} \mid Y>0\right]\right\}=\frac{\alpha}{B}\left\{1-\frac{\lambda}{\lambda+\frac{1}{B s}+\eta}\right\}
$$

Now we let $\eta \downarrow 0$ and $s \downarrow 0$ to conclude that $\alpha \geq 1$. This completes the proof.

\section{Acknowledgements}

This work was supported in part by NSERC of Canada. The work of RvdH and GS was carried out in part at Microsoft Research, and the work of $\mathrm{RvdH}$ and $\mathrm{FdH}$ was carried out in part at the University of British Columbia. We thank Antal Járai and Akira Sakai for stimulating conversations during the initial stages of this work.

\section{References}

[1] M. Aizenman. On the number of incipient spanning clusters. Nucl. Phys. B [FS], 485:551-582, (1997).

[2] M. Aizenman and C.M. Newman. Tree graph inequalities and critical behavior in percolation models. J. Stat. Phys., 36:107-143, (1984).

[3] C. Bezuidenhout and G. Grimmett. The critical contact process dies out. Ann. Probab., 18:1462-1482, (1990).

[4] P. Billingsley. Probability and Measure. John Wiley and Sons, New York, 3rd edition, (1995).

[5] C. Borgs, J.T. Chayes, H. Kesten, and J. Spencer. The birth of the infinite cluster: finite-size scaling in percolation. Commun. Math. Phys., 224:153-204, (2001).

[6] D.C. Brydges and T. Spencer. Self-avoiding walk in 5 or more dimensions. Commun. Math. Phys., 97:125-148, (1985). 
[7] J.T. Chayes and L. Chayes. Percolation and random media. In: K. Osterwalder and R. Stora, editors, Critical Phenomena, Random Systems, Gauge Theories (Les Houches 1984), NorthHolland, Amsterdam, (1986).

[8] J.T. Chayes, L. Chayes, and R. Durrett. Inhomogeneous percolation problems and incipient infinite clusters. J. Phys. A: Math. Gen., 20:1521-1530, (1987).

[9] E.B. Dynkin. Representation for functionals of superprocesses by multiple stochastic integrals, with applications to self-intersection local times. Astérisque, 157-158:147-171, (1988).

[10] G. Grimmett and P. Hiemer. Directed percolation and random walk. In: V. Sidoravicius, editor, In and Out of Equilibrium, pages 273-297. Birkhäuser, Boston, (2002).

[11] T. Hara and G. Slade. The scaling limit of the incipient infinite cluster in high-dimensional percolation. II. Integrated super-Brownian excursion. J. Math. Phys., 41:1244-1293, (2000).

[12] R. van der Hofstad, F. den Hollander, and G. Slade. The survival probability of critical spread-out oriented percolation above $4+1$ dimensions. In preparation.

[13] R. van der Hofstad and G. Slade. A generalised inductive approach to the lace expansion. Probab. Th. Rel. Fields, 122:389-430, (2002).

[14] R. van der Hofstad and G. Slade. Convergence of critical oriented percolation to superBrownian motion above $4+1$ dimensions. Preprint, (2001).

[15] A. Járai Jr. Incipient infinite percolation clusters in 2d. To appear in Ann. Probab.

[16] A. Járai Jr. Invasion percolation and the incipient infinite cluster in 2d. Preprint, (2001).

[17] H. Kesten. The incipient infinite cluster in two-dimensional percolation. Probab. Th. Rel. Fields, 73:369-394, (1986).

[18] J.-F. Le Gall. Spatial Branching Processes, Random Snakes, and Partial Differential Equations. Birkhäuser, Basel, (1999).

[19] N. Madras and G. Slade. The Self-Avoiding Walk. Birkhäuser, Boston, (1993).

[20] B.G. Nguyen and W-S. Yang. Triangle condition for oriented percolation in high dimensions. Ann. Probab., 21:1809-1844, (1993).

[21] B.G. Nguyen and W-S. Yang. Gaussian limit for critical oriented percolation in high dimensions. J. Stat. Phys., 78:841-876, (1995).

[22] E. Perkins. Dawson-Watanabe superprocesses and measure-valued diffusions. In: P.L. Bernard, editor, Lectures on Probability Theory and Statistics. Ecole d'Eté de Probabilités de Saint-Flour XXIX-1999, Springer. To appear.

[23] B. Simon. Functional Integration and Quantum Physics. Academic Press, New York, (1979).

[24] G. Slade. The scaling limit of self-avoiding random walk in high dimensions. Ann. Probab., 17:91-107, (1989). 